\title{
A Novel Three-Component Reaction of Allenoates, Isocyanides and Carboxylic Acids: Facile Synthesis of Highly Substitued Acryl Imide Derivatives
}

\author{
Xian Huang* ${ }^{\text {a, }}$, Feng Sha ${ }^{\mathrm{a}}$
}

a. Department of Chemistry, Zhejiang University (Xixi Campus), Hangzhou 310028,

P. R. China

b. State Key Laboratory of Organometallic Chemistry, Shanghai Institute of Organic Chemistry, Chinese Academy of Sciences, Shanghai 200032, P. R. China

huangx@mail.hz.zj.cn

\section{Supporting Information \\ List of contents}

Experiment procedures..................................S2-S3

Characterization data:

4a-4o and 5, 6

10a-10b, 11a-11h, 13a-13c and 14a-14c

S13-S19

${ }^{1} \mathrm{H}$ NMR and ${ }^{13} \mathrm{C}$ NMR spectra...............................S20-S84

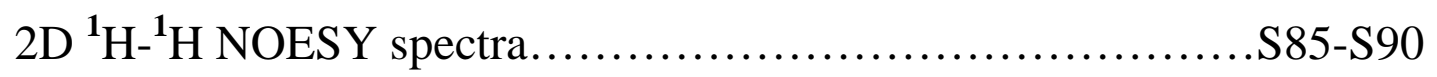




\section{Experiment procedures}

All reactions were performed under an $\mathrm{N}_{2}$ atmosphere. Dry solvents were distilled prior to use: THF and toluene were distilled from sodium-benzophenone; MeCN were distilled from $\mathrm{P}_{2} \mathrm{O}_{5} ; \mathrm{CH}_{2} \mathrm{Cl}_{2}$ were distilled from $\mathrm{CaH}_{2}$. Petroleum ether refers to the fraction with boiling point in the range $60-90{ }^{\circ} \mathrm{C}$. All ${ }^{1} \mathrm{H}$ NMR and ${ }^{13} \mathrm{C}$ NMR spectra were measured in $\mathrm{CDCl}_{3}$ with TMS as the internal standard. Chemical shifts are expressed in ppm and $J$ values are given in Hz. Melting points are uncorrected. Allenoates 1 were prepared according to the known method by treatment of the acid chlorides with the corresponding ethyl 2-(triphenylphoranylidene) propionate. ${ }^{1}$

\section{General Procedure.}

\section{Three-Component Reaction of allenoates with isocyanides and carboxylic acids leading to highly substituted acryl imides 4}

To a stirred solution of allenoate $1(1.0 \mathrm{mmol})$ and isocyanide $2(1.0 \mathrm{mmol})$ in dry MeCN (2 mL) was added a solution of carboxylic acid 3 (1.0 mmol) in dry MeCN (3 $\mathrm{mL}$ ). The reaction mixture was then allowed to stir at $65{ }^{\circ} \mathrm{C}$ for $5 \mathrm{~h}$. The solvent was removed under reduced pressure, and the residue was separated by silica gel chromatography (petroleum ether - ethyl acetate 7:1 v/v) to afford 4.

\subsection{Reaction of allenoic acids and isocyanides leading to polysubstituted}

\section{5-imino-furan-2-ones 10 (a, b) and 13 (a-c). (Condition 1)}

A mixture of allenoic acid $9(1.0 \mathrm{mmol})$ and isocyanide $2(1.0 \mathrm{mmol})$ in dry MeCN (15 mL) was stirred at room temperature for $27 \mathrm{~h}$. The solvent was removed under reduced pressure, and the residue was separated by silica gel chromatography using petroleum ether - ethyl acetate mixture as eluent.

2.2 Reaction of allenoic acids and isocyanides leading to polysubstituted pyrrolidine-2,5-diones 11 (a-h) and 14 (a-c). (Condition 2) 
To a stirred solution of allenoic acid $\mathbf{9}(1.0 \mathrm{mmol})$ and isocyanide $\mathbf{2}(1.0 \mathrm{mmol})$ in dry MeCN (4 mL) was added a mixture of trifluoroacetic acid (TFA, $0.15 \mathrm{mmol}$ ) in dry MeCN ( $1 \mathrm{~mL})$. The reaction mixture was then allowed to stir at $65{ }^{\circ} \mathrm{C}$ for $4 \mathrm{~h}$. Then, the reaction mixture was washed with $5 \%$ aqueous solution of $\mathrm{NaHCO}_{3}(10 \mathrm{~mL})$ and extracted with $\mathrm{Et}_{2} \mathrm{O}(3 \times 10 \mathrm{~mL})$. The combined extracts were dried $\left(\mathrm{Mg}_{2} \mathrm{SO}_{4}\right)$ and concentrated in vacuo. The residue was purifed on silica chromatography using petroleum ether - ethyl acetate mixture as eluent.

\section{Reference:}

1. Lang, R. W.; Hansen, H. J. Org. Synth. 1984, 62, 202. 


\section{Characterization data:}

\section{$2.14 a-40$ and 5,6}

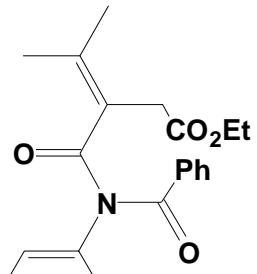

3-(benzoyl-p-tolyl-aminocarbonyl)-4-methyl-pent-3-enoic acid ethyl ester (4a) oil. ${ }^{1} \mathrm{H}$ NMR (400 MHz, $\mathrm{CDCl}_{3}$ ): 7.67-7.64 (m, 2H), 7.41 (t, $\left.J=7.2 \mathrm{~Hz}, 1 \mathrm{H}\right), 7.32$ (t, $J=7.2 \mathrm{~Hz}, 2 \mathrm{H}), 7.12(\mathrm{~d}, J=8.4 \mathrm{~Hz}, 2 \mathrm{H}), 7.06$ (d, $J=8.4 \mathrm{~Hz}, 2 \mathrm{H}), 4.14$ (q, $J=7.2 \mathrm{~Hz}$, 2H), 3.19 (s, 2H), 2.30 (s, 3H), 2.00 (s, 3H), 1.72 (s, 3H), 1.25 (t, J = $7.2 \mathrm{~Hz}, 3 \mathrm{H}) ;{ }^{13} \mathrm{C}$ NMR (100 MHz, $\left.\mathrm{CDCl}_{3}\right): 173.1,172.7,170.5,142.8,137.6,136.4,134.9,131.9$, 129.8, 129.1, 128.2, 127.6, 124.8, 60.8, 36.2, 22.8, 21.4, 21.0, 14.1; IR (neat): 3041, 2984, 1734, 1691, 1658, 1269, $1173 \mathrm{~cm}^{-1}$; MS: m/z (\%) 380 [(M+1)+, 9], 169 (100); HRMS (EI) Calcd for $\mathrm{C}_{23} \mathrm{H}_{25} \mathrm{NO}_{4}$ : 379.1784. Found: 379.1771 .

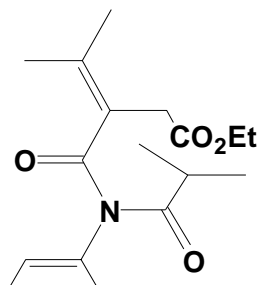

3-(isobutyryl-p-tolyl-aminocarbonyl)-4-methyl-pent-3-enoic acid ethyl ester (4b) oil. ${ }^{1} \mathrm{H}$ NMR (400 MHz, $\mathrm{CDCl}_{3}$ ): 7.21 (d, $\left.J=7.6 \mathrm{~Hz}, 2 \mathrm{H}\right), 7.04$ (d, $J=7.6 \mathrm{~Hz}, 2 \mathrm{H}$ ), 4.12 (q, $J=7.2 \mathrm{~Hz}, 2 \mathrm{H}$ ), 3.18 (s, 2H), 2.96-2.90 (m, 1H), 2.38 (s, 3H), 1.90 (s, 3H), 1.74 (s, 3H), 1.24 (t, $J=7.2 \mathrm{~Hz}, 3 \mathrm{H}), 1.13$ (d, $J=6.8 \mathrm{~Hz}, 6 \mathrm{H}) ;{ }^{13} \mathrm{C}$ NMR (100 MHz, $\left.\mathrm{CDCl}_{3}\right):$ 180.7, 172.7, 170.6, 139.1, 138.4, 135.9, 130.0, 128.2, 125.5, 60.8, 36.1, 34.6, 22.7, 21.2, 20.9, 19.4, 14.2; IR (KBr): 3037, 2968, 2922, 1719, 1690, 1671, 1600, 1514, 1097, 815, $515 \mathrm{~cm}^{-1}$; MS: m/z (\%) 345 (M+1, 7), 169 (100); HRMS (EI) Calcd for $\mathrm{C}_{20} \mathrm{H}_{27} \mathrm{NO}_{4}$ : 345.1940. Found: 345.1943. 


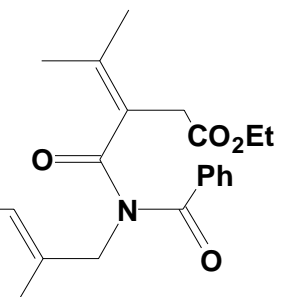

3-(benzoyl-benzyl-aminocarbonyl)-4-methyl-pent-3-enoic acid ethyl ester (4c)

oil. ${ }^{1} \mathrm{H}$ NMR (400 MHz, $\left.\mathrm{CDCl}_{3}\right): 7.49$ (d, $\left.J=6.8 \mathrm{~Hz}, 2 \mathrm{H}\right), 7.42-7.36(\mathrm{~m}, 3 \mathrm{H})$, 7.32-7.28 (m, 3H), 7.28-7.24 (m, 2H), 5.08 (s, 2H), 4.08 (q, J = 7.2 Hz, 2H), 2.67 (s, 2H), 1.70 (s, 3H), 1.34 (s, 3H), 1.21 (t, $J=7.2 \mathrm{~Hz}, 3 \mathrm{H}) ;{ }^{13} \mathrm{C}$ NMR (100 MHz, $\mathrm{CDCl}_{3}$ ): 173.6, 173.5, 170.0, 143.3, 137.4, 137.1, 131.7, 129.0, 128.4, 128.3, 127.5, 127.1, 125.8, 60.8, 48.7, 36.3, 22.7, 21.2, 14.0; IR (KBr): 3032, 2983, 1738, 1696, 1655, 1323, 1177, $701 \mathrm{~cm}^{-1}$; MS: m/z (\%) 379 (M+14), 91 (100); HRMS (EI) Calcd for $\mathrm{C}_{23} \mathrm{H}_{25} \mathrm{NO}_{4}$ : 379.1784. Found: 379.1784.

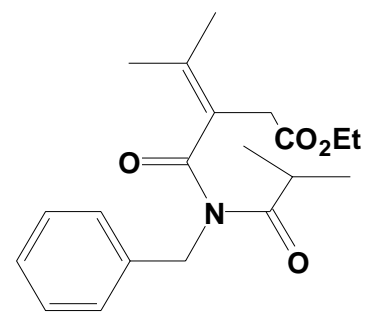

3-(benzyl-isobutyryl-aminocarbonyl)-4-methyl-pent-3-enoic acid ethyl ester (4d) oil. ${ }^{1} \mathrm{H}$ NMR (400 MHz, $\mathrm{CDCl}_{3}$ ): 7.30-7.26 (m, 2H), 7.24-7.20 (m, 3H), 4.90 (s, 2H), 4.08 (q, $J=7.2 \mathrm{~Hz}, 2 \mathrm{H}), 3.30-3.26$ (m, 1H), 3.22 (s, 2H), 1.78 (s, 3H), 1.65 (s, 3H), 1.21 (t, $J=7.2 \mathrm{~Hz}, 3 \mathrm{H}), 1.12$ (d, $J=7.2 \mathrm{~Hz}, 6 \mathrm{H}) ;{ }^{13} \mathrm{C}$ NMR (100 MHz, $\mathrm{CDCl}_{3}$ ): 181.4, 173.9, 170.1, 140.5, 137.6, 128.3, 127.2, 127.1, 124.7, 60.8, 48.0, 35.9, 35.2, 22.1, 21.0, 19.5, 13.9; IR (neat): 3036, 2980, 2936, 1736, 1697, 1652, 1342, 1178, 1090 $\mathrm{cm}^{-1}$; MS: m/z (\%) $345\left(\mathrm{M}^{+}, 9\right), 169$ (100); HRMS (EI) Calcd for $\mathrm{C}_{20} \mathrm{H}_{27} \mathrm{NO}_{4}$ : 345.1940. Found: 345.1948. 


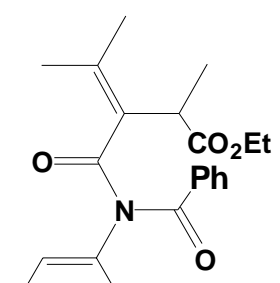

3-(benzoyl-p-tolyl-aminocarbonyl)-2,4-dimethyl-pent-3-enoic acid ethyl ester (4e) oil. ${ }^{1} \mathrm{H}$ NMR (400 MHz, $\mathrm{CDCl}_{3}$ ): 7.57 (d, $\left.J=7.2 \mathrm{~Hz}, 2 \mathrm{H}\right), 7.34-7.31$ (m, 1H), 7.24 (d, $J=7.2 \mathrm{~Hz}, 2 \mathrm{H}), 7.09-7.03$ (m, 4H), 4.09 (q, $J=7.2 \mathrm{~Hz}, 2 \mathrm{H}), 3.71$ (q, $J=7.2 \mathrm{~Hz}, 1 \mathrm{H}$ ), 2.26 (s, 3H), 1.95 (s, 3H), 1.78 (s, 3H), 1.39 (d, $J=7.2 \mathrm{~Hz}, 3 \mathrm{H}), 1.22$ (t, $J=7.2 \mathrm{~Hz}$, 3H); ${ }^{13} \mathrm{C}$ NMR (100 MHz, $\left.\mathrm{CDCl}_{3}\right): 173.9,172.7,171.6,137.5,136.3,134.7,134.5$, 131.5, 130.5, 129.7, 129.5, 128.2, 127.9, 60.6, 41.3, 22.9, 20.9, 20.4, 16.3, 14.0; IR (neat): 3031, 2984, 2926, 1731, 1693, 1671, 1601, 1512, 1450, 1263, $1185 \mathrm{~cm}^{-1}$; MS: m/z (\%) 393 (M+, 5), 183 (100); HRMS (EI) Calcd for $\mathrm{C}_{24} \mathrm{H}_{27} \mathrm{NO}_{4}$ : 393.1940. Found: 393.1951.

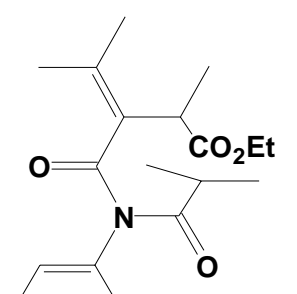

3-(isobutyryl-p-tolyl-aminocarbonyl)-2,4-dimethyl-pent-3-enoic acid ethyl ester (4f)

oil. ${ }^{1} \mathrm{H}$ NMR (400 MHz, $\mathrm{CDCl}_{3}$ ): 7.25 (d, $\left.J=8.0 \mathrm{~Hz}, 2 \mathrm{H}\right), 7.12$ (d, $\left.J=8.0 \mathrm{~Hz}, 2 \mathrm{H}\right)$, 4.15-4.05 (m, 2H), 3.70 (q, J = 7.2 Hz, 1H), 2.61-2.55 (m, 1H), 2.39 (s, 3H), 1.79 (s, 3H), 1.76 (s, 3H), 1.33 (d, $J=7.2 \mathrm{~Hz}, 3 \mathrm{H}), 1.25$ (t, $J=7.2 \mathrm{~Hz}, 3 \mathrm{H}), 1.04$ (d, $J=6.4$ $\mathrm{Hz}, 3 \mathrm{H}), 1.01$ (d, $J=6.4 \mathrm{~Hz}, 3 \mathrm{H}) ;{ }^{13} \mathrm{C} \mathrm{NMR}\left(100 \mathrm{MHz}, \mathrm{CDCl}_{3}\right): 179.2,174.2,172.5$, 138.5, 136.0, 131.7, 130.2, 130.1, 128.5, 60.5, 41.4, 34.3, 22.3, 21.1, 19.8, 19.6, 19.1, 14.1; IR (neat): 3035, 2980, 2934, 1733, 1711, 1696, 1511, 1382, 1302, $1202 \mathrm{~cm}^{-1}$; 
MS: m/z (\%) $359\left(\mathrm{M}^{+}, 6\right), 183$ (100); HRMS (EI) Calcd for $\mathrm{C}_{21} \mathrm{H}_{29} \mathrm{NO}_{4}$ : 359.2097. Found: 359.2091.

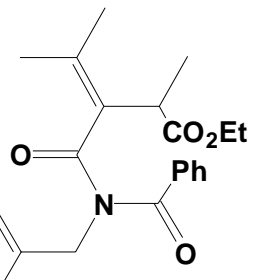

3-(benzoyl-benzyl-aminocarbonyl)-2,4-dimethyl-pent-3-enoic acid ethyl ester (4g) oil. ${ }^{1} \mathrm{H}$ NMR (400 MHz, $\mathrm{CDCl}_{3}$ ): 7.49-7.45 (m, 3H), 7.38-7.35 (m, 2H), 7.25-7.19 (m, 5H), 5.19-4.97 (m, 2H), 4.09 (q, $J=7.2 \mathrm{~Hz}, 2 \mathrm{H}), 3.46$ (q, $J=7.6 \mathrm{~Hz}, 1 \mathrm{H}), 1.72$ (s, 3H), 1.65 (s, 3H), 1.27 (d, $J=7.6 \mathrm{~Hz}, 3 \mathrm{H}), 1.19$ (t, $J=7.2 \mathrm{~Hz}, 3 \mathrm{H}) ;{ }^{13} \mathrm{C}$ NMR (100 $\left.\mathrm{MHz}, \mathrm{CDCl}_{3}\right)$ : 173.8, 173.7, 172.7, 137.2, 135.8, 131.7, 130.5, 128.4, 128.3, 128.1, 128.0, 127.4, 127.3, 60.7, 49.2, 41.2, 23.1, 20.5, 16.2, 14.1; IR (KBr): 3029, 2982, 2938, 1731, 1693, 1657, 1449, 1344, 1199, $729 \mathrm{~cm}^{-1}$; MS: m/z (\%) 393 (M+ 4), 292 (100); HRMS (EI) Calcd for $\mathrm{C}_{24} \mathrm{H}_{27} \mathrm{NO}_{4}$ : 393.1940. Found: 393.1941.

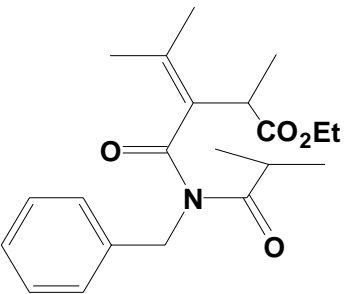

3-(benzyl-isobutyryl-aminocarbonyl)-2,4-dimethyl-pent-3-enoic acid ethyl ester (4h)

m.p. 33-35 ${ }^{\circ} \mathrm{C} .{ }^{1} \mathrm{H}$ NMR (400 MHz, $\mathrm{CDCl}_{3}$ ): 7.31-7.22 (m, 5H), 4.99 (s, 2H), 4.15-4.11 (m, 2H), 3.57 (q, $J=7.2 \mathrm{~Hz}, 1 \mathrm{H}), 3.37-3.31$ (m, 1H), 1.75 (s, 3H), 1.56 (s, 3H), 1.37 (d, $J=7.2 \mathrm{~Hz}, 3 \mathrm{H}), 1.21$ (t, $J=7.2 \mathrm{~Hz}, 3 \mathrm{H}), 1.12$ (d, $J=7.2 \mathrm{~Hz}, 3 \mathrm{H}), 1.10$ (d, $J=7.2 \mathrm{~Hz}, 3 \mathrm{H}) ;{ }^{13} \mathrm{C}$ NMR (100 MHz, $\left.\mathrm{CDCl}_{3}\right):$ 181.3, 173.5, 173.4, 137.7, 131.1, 128.3, 128.2, 127.2, 127.0, 60.8, 47.9, 41.2, 35.4, 22.3, 20.4, 19.7, 19.3, 16.5, 14.0; IR (KBr): 3032, 2981, 2937, 1734, 1691, 1652, 1382, 1350, 1183, $737 \mathrm{~cm}^{-1}$; MS: m/z (\%) 359 ( $\left.\mathrm{M}^{+}, 4\right), 91$ (100); HRMS (EI) Calcd for $\mathrm{C}_{21} \mathrm{H}_{29} \mathrm{NO}_{4}$ : 359.2097. Found: 359.2104. 


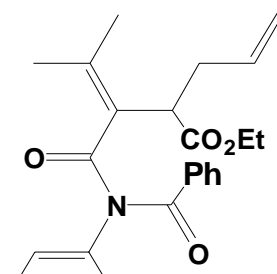

2-allyl-3-(benzoyl-p-tolyl-aminocarbonyl)-4-methyl-pent-3-enoic acid ethyl ester (4i)

oil. ${ }^{1} \mathrm{H}$ NMR (400 MHz, $\mathrm{CDCl}_{3}$ ): 7.53 (d, $\left.J=7.2 \mathrm{~Hz}, 2 \mathrm{H}\right), 7.32-7.28$ (m, 1H), 7.19 (d, $J=7.2 \mathrm{~Hz}, 2 \mathrm{H}), 7.08-7.03$ (m, 4H), 5.91-5.78 (m, 1H), 5.11-4.99 (m, 2H), 4.10 (q, $J=$ 7.6 Hz, 2H), 3.73-3.69 (m, 1H), 2.74-2.70 (m, 1H), 2.48-2.44 (m, 1H), 2.24 (s, 3H), 1.94 (s, 3H), 1.79 (s, 3H), 1.23 (t, $J=7.6 \mathrm{~Hz}, 3 \mathrm{H}) ;{ }^{13} \mathrm{C}$ NMR (100 MHz, $\left.\mathrm{CDCl}_{3}\right)$ : 172.8, 172.4, 171.3, 137.5, 136.1, 135.5, 134.4, 131.4, 129.6, 129.4, 129.3, 129.0, 128.1, 127.8, 116.7, 60.5, 47.5, 36.1, 22.8, 20.9, 20.1, 14.1; IR (KBr): 3062, 2981, 2921, 1731, 1694, 1652, 1511, 1448, 1270, 1178, $710 \mathrm{~cm}^{-1}$; MS: m/z (\%) $419\left(\mathrm{M}^{+}, 6\right)$, 209 (100); HRMS (EI) Calcd for $\mathrm{C}_{26} \mathrm{H}_{29} \mathrm{NO}_{4}$ : 419.2097. Found: 419.2103.

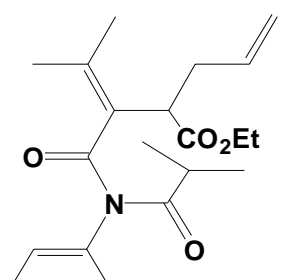

2-allyl-3-(isobutyryl-p-tolyl-aminocarbonyl)-4-methyl-pent-3-enoic acid ethyl ester $(4 \mathbf{j})$

oil. ${ }^{1} \mathrm{H}$ NMR (400 MHz, $\mathrm{CDCl}_{3}$ ): 7.27 (d, $\left.J=8.0 \mathrm{~Hz}, 2 \mathrm{H}\right), 7.15$ (d, $\left.J=8.0 \mathrm{~Hz}, 2 \mathrm{H}\right)$, 5.85-5.73 (m, 1H), 5.11-4.97 (m, 2H), 4.12-4.08 (m, 2H), 3.71-3.67 (m, 1H), 2.62-2.47 (m, 2H), 2.45-2.38 (m, 1H), 2.42 (s, 3H), 1.77 (s, 6H), 1.27 (t, J = 7.2 Hz, 3H), 1.03 (d, $J=6.8 \mathrm{~Hz}, 3 \mathrm{H}), 0.97$ (d, $J=6.8 \mathrm{~Hz}, 3 \mathrm{H})$; 
${ }^{13} \mathrm{C}$ NMR (100 MHz, $\mathrm{CDCl}_{3}$ ): 178.8, 173.2, 172.2, 138.5, 135.9, 135.3, 130.2, 130.1, 128.4, 128.2, 116.8, 60.4, 47.4, 36.6, 34.1, 22.1, 21.1, 19.7, 18.8, 14.2; IR (neat): 3039, 2981, 2934, 1732, 1712, 1694, 1512, 1264, 1199, $1164 \mathrm{~cm}^{-1}$; MS: m/z (\%) $385\left(\mathrm{M}^{+}\right.$, 11), 209 (100); Anal. Calcd for $\mathrm{C}_{23} \mathrm{H}_{31} \mathrm{NO}_{4}$ (385.23): C 71.66, H 8.11, N 3.63. Found: C 71.65, H 8.05, N 3.56.

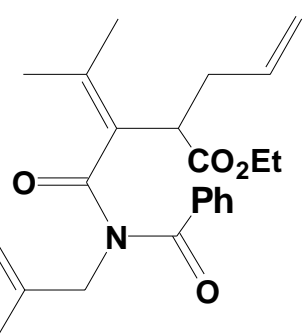

2-allyl-3-(benzoyl-benzyl-aminocarbonyl)-4-methyl-pent-3-enoic acid ethyl ester (4k)

oil. ${ }^{1} \mathrm{H}$ NMR (400 MHz, $\left.\mathrm{CDCl}_{3}\right)$ : 7.48-7.44 (m, 3H), 7.38-7.34 (m, 2H), 7.23-7.16 (m, 5H), 5.85-5.74 (m, 1H), 5.11-4.99 (m, 4H), 4.06 (q, $J=7.2 \mathrm{~Hz}, 2 \mathrm{H}), 3.61-3.54$ (m, 1H), 2.73-2.63 (m, 1H), 2.37-2.27 (m, 1H), 1.71 (s, 3H), 1.70 (s, 3H), 1.20 (t, $J=7.2$ $\mathrm{Hz}, 3 \mathrm{H}) ;{ }^{13} \mathrm{C}$ NMR (100 MHz, $\left.\mathrm{CDCl}_{3}\right)$ : 173.5, 172.7, 172.3, 137.0, 135.5, 135.4, 131.6, 129.7, 129.0, 128.7, 128.3, 128.0, 127.9, 127.2, 116.8, 60.6, 49.2, 47.3, 36.0, 23.0, 20.2, 14.1; IR (KBr): 3064, 2981, 1732, 1683, 1655, 1448, 1345, 1219, 1178, 961, $700 \mathrm{~cm}^{-1}$; MS: m/z (\%) $419\left(\mathrm{M}^{+}, 24\right), 209$ (100); HRMS (EI) Calcd for $\mathrm{C}_{26} \mathrm{H}_{29} \mathrm{NO}_{4}$ : 419.2097. Found: 419.2101.

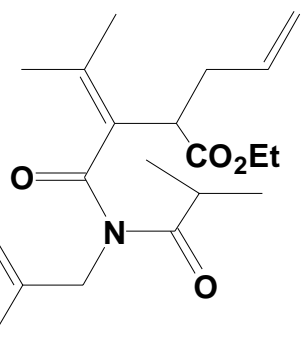

2-allyl-3-(benzyl-isobutyryl-aminocarbonyl)-4-methyl-pent-3-enoic acid ethyl ester (4l) 
m.p. 29-31 ${ }^{\circ} \mathrm{C} .{ }^{1} \mathrm{H}$ NMR (400 MHz, $\mathrm{CDCl}_{3}$ ): 7.32-7.20 (m, 5H), 5.87-5.71 (m, 1H), 5.11-4.94 (m, 2H), 4.89 (s, 2H), 4.15-4.05 (m, 2H), 3.62-3.59 (m, 1H), $3.22(\mathrm{~s}, 1 \mathrm{H})$, 2.75-2.67 (m, 1H), 2.45-2.38 (m, 1H), 1.76 (s, 3H), 1.50 (s, 3H), 1.23 (t, J = 7.2 Hz, 3H), 1.13 (d, $J=6.4 \mathrm{~Hz}, 3 \mathrm{H}), 1.07$ (s, 3H); ${ }^{13} \mathrm{C} \mathrm{NMR}\left(100 \mathrm{MHz}, \mathrm{CDCl}_{3}\right.$ ): 180.9, 173.1, 172.7, 137.7, 135.3, 129.5, 128.4, 128.3, 127.1, 127.0, 116.9, 60.8, 47.8, 47.3, 36.3, 35.2, 22.5, 20.3, 19.9, 14.1; IR (KBr): 3061, 2979, 2936, 1733, 1689, 1652 , 1384, 1346, 1185, $735 \mathrm{~cm}^{-1}$; MS: m/z (\%) 385 (M+1 8), 91 (100); Anal. Calcd for $\mathrm{C}_{23} \mathrm{H}_{31} \mathrm{NO}_{4}$ (385.23): C 71.66, H 8.11, N 3.63. Found: C 71.69, H 8.13, N 3.54.

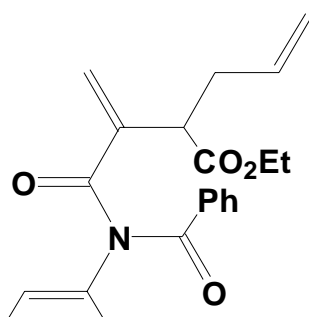

2-[1-(benzoyl-p-tolyl-aminocarbonyl)-vinyl]-pent-4-enoic acid ethyl ester (4m)

${ }^{1} \mathrm{H}$ NMR (400 MHz, $\mathrm{CDCl}_{3}$ ): 7.53 (d, $\left.J=8.0 \mathrm{~Hz}, 2 \mathrm{H}\right), 7.36-7.34$ (m, 2H), 7.22-7.18 (m, 3H), 7.07 (d, $J=8.0 \mathrm{~Hz}, 2 \mathrm{H}), 6.39$ (s, 1H), 5.89 (s, 1H), 5.83-5.73 (m, 1H), 5.19-5.07 (m, 2H), 4.23 (q, $J=7.2 \mathrm{~Hz}, 2 \mathrm{H}), 3.59-3.52$ (m, 1H), 2.85-2.78 (m, 1H), 2.42-2.31 (m, 1H), 2.27 (s, 3H), 1.30 (t, $J=7.2 \mathrm{~Hz}, 3 \mathrm{H})$.

2-[2-(benzoyl-p-tolyl-amino)-1-methyl-2-oxo-ethylidene]-pent-4-enoic acid ethyl ester (5) 
${ }^{1} \mathrm{H}$ NMR (400 MHz, $\mathrm{CDCl}_{3}$ ): 7.53 (d, $\left.J=8.0 \mathrm{~Hz}, 2 \mathrm{H}\right), 7.31-7.28$ (m, 1H), 7.22-7.19 (m, 4H), 7.07 (d, $J=8.0 \mathrm{~Hz}, 2 \mathrm{H}), 5.19-5.67$ (m, 1H), 5.01-4.96 (m, 2H), 4.16 (q, $J=$ $7.2 \mathrm{~Hz}, 2 \mathrm{H}$ ), 3.06 (d, $J=6.8 \mathrm{~Hz}, 2 \mathrm{H}), 2.25$ (s, 3H), 2.15 (s, 3H), 1.20 (t, $J=7.2 \mathrm{~Hz}$, $3 \mathrm{H})$.

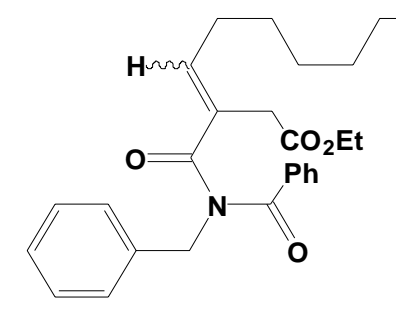

(E)-3-(enzoyl-benzyl-aminocarbonyl)-undec-3-enoic acid ethyl ester [4n (E-isomer)]

oil. ${ }^{1} \mathrm{H}$ NMR (400 MHz, $\mathrm{CDCl}_{3}$ ): 7.47-7.42 (m, 5H), 7.33-7.27 (m, 5H), 6.09 (t, $J=$ $7.2 \mathrm{~Hz}, 1 \mathrm{H}), 5.08$ (s, 2H), 4.06 (q, $J=7.2 \mathrm{~Hz}, 2 \mathrm{H}), 2.88$ (s, 2H), 1.87-1.80 (m, 2H), 1.28-1.17 (m, 6H), 1.23 (t, $J=7.2 \mathrm{~Hz}, 3 \mathrm{H}), 1.16-1.08$ (m, 4H), 0.88 (t, $J=7.2 \mathrm{~Hz}$, 3H); ${ }^{13} \mathrm{C}$ NMR (100 MHz, $\mathrm{CDCl}_{3}$ ): 174.6, 173.1, 169.6, 145.1, 137.4, 137.1, 131.7, 131.5, 128.5, 128.3, 128.2, 127.9, 127.3, 60.7, 49.8, 39.8, 33.2, 31.4, 29.0, 28.8, 28.7, 27.8, 22.4, 13.9; IR (KBr): 3057, 2979, 2936, 2875, 1736, 1697, 1656, 1497, 1385, 1186, 1145, 742, 721, $700 \mathrm{~cm}^{-1}$; MS: m/z (\%) $449\left(\mathrm{M}^{+}, 4\right), 91$ (100); Anal. Calcd for $\mathrm{C}_{28} \mathrm{H}_{35} \mathrm{NO}_{4}$ (449.26): C 74.80, H 7.85, N 3.12. Found: C 74.91, H 7.72, N 3.11.

(Z)-3-(enzoyl-benzyl-aminocarbonyl)-undec-3-enoic acid ethyl ester [4n (Z-isomer)]

${ }^{1} \mathrm{H}$ NMR (400 MHz, $\mathrm{CDCl}_{3}$ ): 7.39-7.30 (m, 5H), 7.25-7.21 (m, 5H), 5.39 (t, $J=7.2$ Hz, 1H), 5.06 (s, 2H), 4.05 (q, $J=7.2 \mathrm{~Hz}, 2 \mathrm{H}), 2.78$ (s, 2H), 2.05-1.97 (m, 2H), 1.25-1.08 (m, 13H), 0.87 (t, $J=7.2 \mathrm{~Hz}, 3 \mathrm{H})$. 


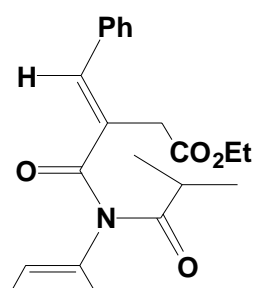

(E)-3-(isobutyryl-p-tolyl-aminocarbonyl)-4-phenyl-but-3-enoic acid ethyl ester (40)

oil. ${ }^{1} \mathrm{H}$ NMR (500 MHz, $\mathrm{CDCl}_{3}$ ): 7.39 (s, 1H), 7.35 (t, $\left.J=7.0 \mathrm{~Hz}, 2 \mathrm{H}\right), 7.31$ (t, $J=7.0$ Hz, 1H), 7.25 (d, $J=7.0 \mathrm{~Hz}, 2 \mathrm{H}), 7.22$ (d, $J=7.5 \mathrm{~Hz}, 2 \mathrm{H}), 7.12$ (d, $J=7.5 \mathrm{~Hz}, 2 \mathrm{H}$ ) 4.18 (q, $J=7.0 \mathrm{~Hz}, 2 \mathrm{H}), 3.48$ (s, 2H), 3.06-2.99 (m, 1H), 2.36 (s, 3H), 1.26 (t, $J=7.0$ $\mathrm{Hz}, 3 \mathrm{H}), 1.21$ (d, $J=7.0 \mathrm{~Hz}, 6 \mathrm{H}) ;{ }^{13} \mathrm{C} \mathrm{NMR}\left(100 \mathrm{MHz}, \mathrm{CDCl}_{3}\right):$ 181.3, 173.9, 170.5, 140.5, 138.0, 136.4, 134.5, 131.1, 130.0, 128.9, 128.7, 128.4, 127.9, 61.0, 34.7, 34.2, 21.0, 19.5, 14.0; IR (KBr): 3019, 2976, 2931, 1732, 1689, 1671, 1511, 1448, 1163, 1027, 810, $698 \mathrm{~cm}^{-1}$; MS: m/z (\%) $393\left(\mathrm{M}^{+}, 13\right), 217$ (100); Anal. Calcd for $\mathrm{C}_{24} \mathrm{H}_{27} \mathrm{NO}_{4}$ (393.48): C 73.26, H 6.92, N 3.56. Found: C 73.22, H 6.95, N 3.49.

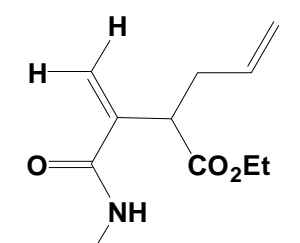

\section{2-(1-p-tolylcarbamoyl-vinyl)-pent-4-enoic acid ethyl ester (6)}

oil. ${ }^{1} \mathrm{H}$ NMR (400 MHz, $\mathrm{CDCl}_{3}$ ): 8.04 (s, 1H), 7.38 (d, $\left.J=7.6 \mathrm{~Hz}, 2 \mathrm{H}\right), 7.09$ (d, $J=$ $7.6 \mathrm{~Hz}, 2 \mathrm{H}), 6.41$ (s, 1H), 5.91 (s, 1H), 5.81-5.72 (m, 1H), 5.14-5.03 (m, 2H), 4.25 (q, $J=7.2 \mathrm{~Hz}, 2 \mathrm{H}), 3.61$ (t, $J=7.6 \mathrm{~Hz}, 1 \mathrm{H}), 2.88-2.78$ (m, 1H), 2.46-2.36 (m, 1H), 2.29 (s, 3H), 1.32 (t, $J=7.2 \mathrm{~Hz}, 3 \mathrm{H}) ;{ }^{13} \mathrm{C}$ NMR (100 MHz, $\left.\mathrm{CDCl}_{3}\right): 169.5,167.7,137.8$, 135.5, 135.2, 133.7, 129.4, 127.4, 119.5, 117.2, 61.6, 47.9, 34.7, 20.8, 14.1; IR (KBr): 3425, 3033, 2980, 2923, 1713, 1691, 1603, 1515, 1250, 1113, $818 \mathrm{~cm}^{-1}$; MS: m/z (\%) 287 ( $\mathrm{M}^{+}$, 43), 107 (100); Anal. Calcd for $\mathrm{C}_{17} \mathrm{H}_{21} \mathrm{NO}_{3}$ (287.15): C 71.06, H 7.37, N 4.87. Found: C 71.13, H 7.49, N 4.78. 
2.2 10a-10b, 11a-11h, 13a-13c and 14a-14c

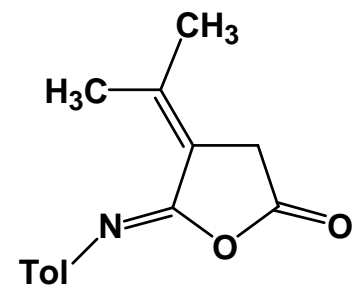

\section{4-isopropylidene-5-p-tolylimino-dihydro-furan-2-one (10a)}

oil. ${ }^{1} \mathrm{H}$ NMR (400 MHz, $\mathrm{CDCl}_{3}$ ): 7.15 (d, $\left.J=8.4 \mathrm{~Hz}, 2 \mathrm{H}\right), 7.09$ (d, $J=8.4 \mathrm{~Hz}, 2 \mathrm{H}$ ), 3.43 (s, 2H), 2.43 (s, 3H), 2.35 (s, 3H), 1.92 (s, 3H); ${ }^{13} \mathrm{C}$ NMR (100 MHz, $\left.\mathrm{CDCl}_{3}\right)$ : 170.5, 148.9, 146.4, 142.2, 134.4, 129.2, 123.0, 117.5, 33.4, 24.7, 21.5, 20.9; IR (KBr): 2911, 1811, 1682, 1657, 1202, 1147, 985, 922, $826 \mathrm{~cm}^{-1}$; MS: m/z (\%) 229 $\left(\mathrm{M}^{+}, 100\right), 67$ (82); HRMS (EI) Calcd for $\mathrm{C}_{14} \mathrm{H}_{15} \mathrm{NO}_{2}$ : 229.1103. Found: 229.1104.

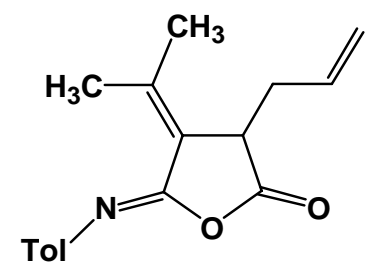

\section{3-allyl-4-isopropylidene-5-p-tolylimino-dihydro-furan-2-one (10b)}

oil. ${ }^{1} \mathrm{H}$ NMR (400 MHz, $\mathrm{CDCl}_{3}$ ): 7.14 (d, $\left.J=8.0 \mathrm{~Hz}, 2 \mathrm{H}\right), 7.08$ (d, $J=8.0 \mathrm{~Hz}, 2 \mathrm{H}$ ), 5.83-5.73 (m, 1H), 5.22-5.15 (m, 2H), 3.64-3.57 (m, 1H), 2.69-2.64 (m, 2H), 2.44 (d, $J=1.6 \mathrm{~Hz}, 3 \mathrm{H}), 2.35$ (s, 3H), 1.99 (s, H); ${ }^{13} \mathrm{C} \mathrm{NMR} \mathrm{(100} \mathrm{MHz,} \mathrm{CDCl}_{3}$ ): 173.1, 148.8, 146.7, 142.3, 134.4, 131.6, 129.2, 123.0, 121.5, 119.6, 44.3, 35.8, 24.0, 21.9, 20.9; IR (KBr): 3064, 2921, 1764, 1660, 1597, 1517, 1406, 1244, 932, $817 \mathrm{~cm}^{-1}$; MS: m/z (\%) 269 ( $\left.\mathrm{M}^{+}, 73\right), 228$ (100); HRMS (EI) Calcd for $\mathrm{C}_{17} \mathrm{H}_{19} \mathrm{NO}_{2}$ : 269.1416. Found: 269.1422.

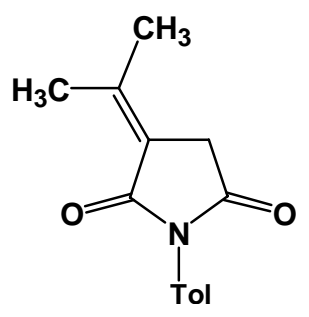

3-isopropylidene-1-p-tolyl-pyrrolidine-2,5-dione (11a) 
m.p. 108-110 ${ }^{\circ} \mathrm{C} .{ }^{1} \mathrm{H}$ NMR (400 MHz, $\mathrm{CDCl}_{3}$ ): 7.26 (d, $\left.J=8.4 \mathrm{~Hz}, 2 \mathrm{H}\right), 7.17$ (d, $J=$ $8.4 \mathrm{~Hz}, 2 \mathrm{H}$ ), 3.37 (s, 2H), 2.37 (s, 6H), 1.92 (s, 3H); ${ }^{13} \mathrm{C}$ NMR (100 MHz, $\mathrm{CDCl}_{3}$ ): 173.0, 168.8, 150.7, 138.3, 129.6, 129.4, 126.4, 118.6, 34.2, 24.2, 21.1, 20.8; IR (KBr): 2919, 1700, 1660, 1515, 1384, 1183, $782 \mathrm{~cm}^{-1}$; MS: m/z (\%) 229 (M+100), 68 (94); HRMS (EI) Calcd for $\mathrm{C}_{14} \mathrm{H}_{15} \mathrm{NO}_{2}$ : 229.1103. Found: 229.1103.

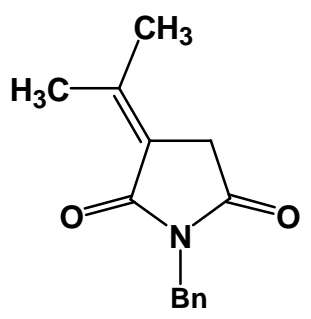

1-benzyl-3-isopropylidene-pyrrolidine-2,5-dione (11b)

m.p. 100-102 ${ }^{\circ} \mathrm{C} .{ }^{1} \mathrm{H}$ NMR (400 MHz, $\mathrm{CDCl}_{3}$ ): 7.41-7.39 (m, 2H), 7.32-7.25 (m, 3H), 4.70 (s, 2H), 3.21 (s, 2H), 3.34 (s, 3H), 1.86 (s, 3H); ${ }^{13} \mathrm{C}$ NMR (100 MHz, CDCl $)$ : 173.5, 169.4, 149.8, 136.2, 128.8, 128.5, 127.7, 118.9, 41.9, 34.1, 24.1, 20.7; IR (KBr): 2954, 1694, 1665, 1395, 1178, $698 \mathrm{~cm}^{-1}$; MS: m/z (\%) 229 (M+, 98), 91 (100); HRMS (EI) Calcd for $\mathrm{C}_{14} \mathrm{H}_{15} \mathrm{NO}_{2}$ : 229.1103. Found: 229.1107.

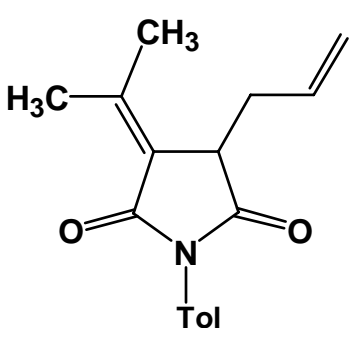

3-allyl-4-isopropylidene-1-p-tolyl-pyrrolidine-2,5-dione (11c)

oil. ${ }^{1} \mathrm{H}$ NMR (400 MHz, CDCl 3 ): 7.24 (d, $\left.J=8.4 \mathrm{~Hz}, 2 \mathrm{H}\right), 7.13$ (d, $\left.J=8.4 \mathrm{~Hz}, 2 \mathrm{H}\right)$, 5.78-5.65 (m, 1H), 5.16-5.08 (m, 2H), 3.61-3.50 (m, 1H), 2.77-2.75 (m, $1 \mathrm{H})$, 2.67-2.65 (m, 2H), 2.38 (s, 3H), 2.37 (s, 3H), 1.98 (s, 3H); ${ }^{13} \mathrm{C}$ NMR (100 MHz, $\left.\mathrm{CDCl}_{3}\right): 175.8,168.7,150.8,138.2,131.7,129.5,129.3,126.4,122.3,119.2,44.0$, 35.1, 23.8, 21.1, 21.0; IR (KBr): 3041, 2922, 1706, 1660, 1515, 1383, 1186, $797 \mathrm{~cm}^{-1}$; MS: m/z (\%) 269 (M+100), 228 (79); HRMS (EI) Calcd for $\mathrm{C}_{17} \mathrm{H}_{19} \mathrm{NO}_{2}$ : 269.1416. Found: 269.1418. 


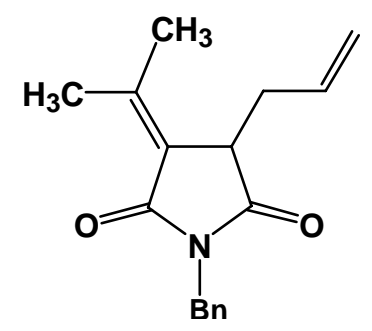

\section{3-allyl-1-benzyl-4-isopropylidene-pyrrolidine-2,5-dione (11d)}

oil. ${ }^{1} \mathrm{H}$ NMR (400 MHz, $\left.\mathrm{CDCl}_{3}\right): 7.37$ (d, $\left.J=6.8 \mathrm{~Hz}, 2 \mathrm{H}\right), 7.31-7.24(\mathrm{~m}, 3 \mathrm{H})$, 5.52-5.42 (m, 1H), 5.01-4.88 (m, 2H), 4.71-4.62 (m, 2H), 3.38 (s, 1H), 2.67-2.65 (m, 1H), 2.55-2.51 (m, 1H), 2.35 (s, 3H), 1.93 (s, 3H); ${ }^{13} \mathrm{C}$ NMR (100 MHz, $\mathrm{CDCl}_{3}$ ): 176.4, 169.3, 150.1, 136.2, 131.7, 128.7, 128.4, 127.6, 122.6, 119.0, 44.0, 41.8, 34.9, 23.8, 21.1; IR (KBr): 3049, 2932, 1698, 1661, 1396, 1181, $701 \mathrm{~cm}^{-1}$; MS: m/z (\%) $269\left(\mathrm{M}^{+}\right.$, 57), 150 (100); HRMS (EI) Calcd for $\mathrm{C}_{17} \mathrm{H}_{19} \mathrm{NO}_{2}$ : 269.1416. Found: 269.1423.

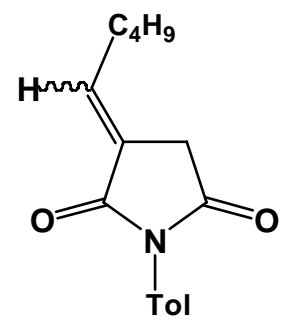

(E)-3-pentylidene-1-p-tolyl-pyrrolidine-2,5-dione [11e ( $E$-isomer)] m.p. 98-100 ${ }^{\circ} \mathrm{C} .{ }^{1} \mathrm{H}$ NMR (400 MHz, $\mathrm{CDCl}_{3}$ ): 7.27 (d, $J=7.2 \mathrm{~Hz}, 2 \mathrm{H}$ ), 7.19 (d, $J=$ $7.2 \mathrm{~Hz}, 2 \mathrm{H}), 6.96-6.91$ (m, 1H), 3.37 (t, $J=1.2 \mathrm{~Hz}, 2 \mathrm{H}$ ), 2.38 (s, 3H), 2.23 (q, $J=7.2$ $\mathrm{Hz}, 2 \mathrm{H}), 1.55-1.45$ (m, 2H), 1.43-1.33 (m, 2H), 0.92 (t, $J=7.2 \mathrm{~Hz}, 3 \mathrm{H}) ;{ }^{13} \mathrm{C} \mathrm{NMR}$ (100 MHz, $\left.\mathrm{CDCl}_{3}\right):$ 173.3, 169.0, 140.0, 138.5, 129.7, 129.3, 126.2, 125.2, 32.0, 30.1, 29.6, 22.3, 21.1, 13.8; IR (KBr): 3042, 2957, 2928, 1708, 1676, 1517, 1386, 1189, 1154, 823, 732, 658, $513 \mathrm{~cm}^{-1}$; MS: m/z (\%) 257 (M+1 100), 228 (50); HRMS (EI) Calcd for $\mathrm{C}_{16} \mathrm{H}_{19} \mathrm{NO}_{2}$ : 257.1416. Found: 257.1412.

\section{(Z)-3-pentylidene-1-p-tolyl-pyrrolidine-2,5-dione [11e (Z-isomer)]}

${ }^{1} \mathrm{H}$ NMR (400 MHz, $\mathrm{CDCl}_{3}$ ): 7.27 (d, $\left.J=7.2 \mathrm{~Hz}, 2 \mathrm{H}\right), 7.19$ (d, $J=7.2 \mathrm{~Hz}, 2 \mathrm{H}$ ), 6.27-6.22 (m, 1H), 3.42 (s, 2H), 2.83 (q, J = 7.2 Hz, 2H), 2.30 (s, 3H), 1.42-1.33 (m, 2H), 1.26-1.20 (m, 2H), $0.92(\mathrm{t}, J=7.2 \mathrm{~Hz}, 3 \mathrm{H})$. 


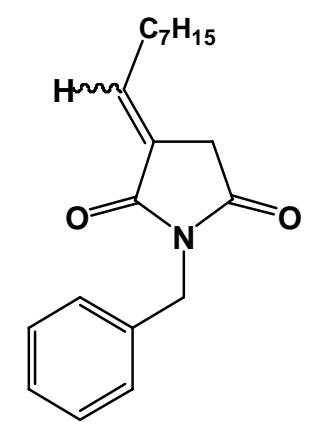

(E)-1-benzyl-3-octylidene-pyrrolidine-2,5-dione [11f ( $E$-isomer)]

oil. ${ }^{1} \mathrm{H}$ NMR (400 MHz, $\mathrm{CDCl}_{3}$ ): 7.43 (d, $\left.J=6.8 \mathrm{~Hz}, 2 \mathrm{H}\right), 7.33-7.25$ (m, 3H), 6.85-6.80 (m, 1H), 4.73 (s, 2H), 3.20 (s, 2H), 2.17 (q, $J=7.2 \mathrm{~Hz}, 2 \mathrm{H}), 1.53-1.44$ (m, 2H), 1.31-1.24 (m, 8H), 0.89 (t, $J=6.8 \mathrm{~Hz}, 3 \mathrm{H}) ;{ }^{13} \mathrm{C}$ NMR (100 MHz, $\left.\mathrm{CDCl}_{3}\right)$ : 173.7, 169.5, 139.1, 135.9, 128.8, 128.5, 127.8, 125.4, 42.2, 31.9, 31.6, 29.8, 29.2, 28.9, 28.0, 22.5, 14.0; IR (KBr): 3051, 2977, 2923, 1715, 1686, 1396, 1183, 712, $675 \mathrm{~cm}^{-1}$; MS: m/z (\%) 299 (M+19), 91 (100); Anal. Calcd for $\mathrm{C}_{19} \mathrm{H}_{25} \mathrm{NO}_{2}$ (299.19): C 76.22, H 8.42, N 4.68. Found: C 76.09, H 8.51, N 4.61.

\section{(Z)-1-benzyl-3-octylidene-pyrrolidine-2,5-dione [11f (Z-isomer)]}

${ }^{1} \mathrm{H}$ NMR (400 MHz, $\mathrm{CDCl}_{3}$ ): 7.43 (d, $J=6.8 \mathrm{~Hz}, 2 \mathrm{H}$ ), 7.33-7.25 (m, 3H), 6.18-6.10 (m, 1H), 4.69 (s, 2H), 3.24 (s, 2H), 2.78 (q, $J=7.2 \mathrm{~Hz}, 2 \mathrm{H}), 1.53-1.44$ (m, 2H), 1.31-1.23 (m, 8H), 0.89 (t, $J=6.8 \mathrm{~Hz}, 3 \mathrm{H})$.

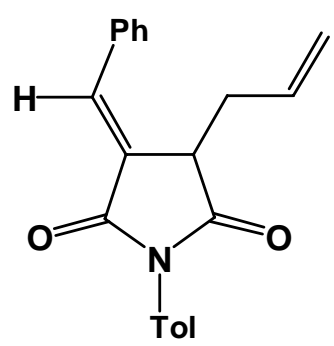

(E)-3-allyl-4-benzylidene-1-p-tolyl-pyrrolidine-2,5-dione (11g)

m.p. $144-146{ }^{\circ} \mathrm{C} .{ }^{1} \mathrm{H}$ NMR (400 MHz, $\mathrm{CDCl}_{3}$ ): 7.80 (d, $J=2.4 \mathrm{~Hz}, 1 \mathrm{H}$ ), 7.56-7.54 (m, 2H), 7.51-7.44 (m, 3H), 7.30 (d, $J=8.0 \mathrm{~Hz}, 2 \mathrm{H}), 7.23$ (d, $J=8.0 \mathrm{~Hz}, 2 \mathrm{H}), 5.73-5.60$ (m, 1H), 5.08-4.99 (m, 2H), 4.09-4.06 (m, 1H), 2.88-2.78 (m, 1H), 2.75-2.65 (m, 1H), 2.41 (s, 3H); ${ }^{13} \mathrm{C}$ NMR (100 MHz, $\left.\mathrm{CDCl}_{3}\right)$ : 176.1, 169.8, 138.6, 135.7, 133.6, 131.4, 130.0, 129.9, 129.7, 129.3, 129.0, 127.2, 126.2, 119.6, 43.1, 32.2, 21.2; 
IR (KBr): 3109, 2922, 1706, 1647, 1514, 1379, 1175, $697 \mathrm{~cm}^{-1}$; MS: m/z (\%) $317\left(\mathrm{M}^{+}\right.$, 12), 115 (100); HRMS (EI) Calcd for $\mathrm{C}_{21} \mathrm{H}_{19} \mathrm{NO}_{2}$ : 317.1416. Found: 317.1413.

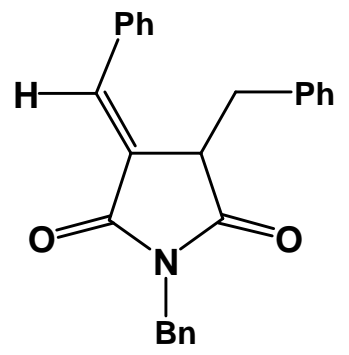

(E)-1,3-dibenzyl-4-benzylidene-pyrrolidine-2,5-dione (11h)

m.p. 97-99 ${ }^{\circ}$ C. ${ }^{1} \mathrm{H}$ NMR (400 MHz, $\mathrm{CDCl}_{3}$ ): 7.65 (d, $\left.J=1.6 \mathrm{~Hz}, 1 \mathrm{H}\right), 7.53$ (d, $J=7.6$ Hz, 2H), 7.48-7.43 (m, 2H), 7.23-7.20 (m, 3H), 7.13-7.10 (m, 2H), 7.06-7.02 (m, 1H), 6.95 (t, $J=7.6 \mathrm{~Hz}, 2 \mathrm{H}), 6.70$ (d, $J=7.6 \mathrm{~Hz}, 2 \mathrm{H}), 4.57$ (s, 3H), 4.09-4.06 (m, 1H), 3.30-3.25 (m, 1H), 3.15-3.10 (m, 1H); ${ }^{13} \mathrm{C} \mathrm{NMR} \mathrm{(100} \mathrm{MHz,} \mathrm{CDCl}_{3}$ ): 176.3, 170.0, 135.4, 135.2, 135.1, 133.7, 130.0, 129.9, 129.3, 129.0, 128.4, 128.3, 128.0, 127.5, 127.3, 126.8, 44.3, 42.2, 32.6; IR (KBr): 3081, 2975, 2922, 1718, 1699, 1603, 1389, $1190 \mathrm{~cm}^{-1}$; MS: m/z (\%) 367 (M+ 28), 91 (100); Anal. Calcd for $\mathrm{C}_{25} \mathrm{H}_{21} \mathrm{NO}_{2}$ (367.16): C 81.72, H 5.76, N 3.81. Found: C 81.79, H 5.83, N 3.75.

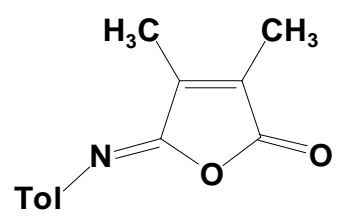

\section{3,4-dimethyl-5-p-tolylimino-5H-furan-2-one (13a)}

m.p. 81-83 ${ }^{\circ}$ C. ${ }^{1} \mathrm{H}$ NMR (400 MHz, $\mathrm{CDCl}_{3}$ ): 7.29 (d, $J=8.0 \mathrm{~Hz}, 2 \mathrm{H}$ ), 7.16 (d, $J=8.0$ Hz, 2H), 2.35 (s, 3H), 2.18 (d, $J=1.2 \mathrm{~Hz}, 3 \mathrm{H}), 2.02$ (d, $J=1.2 \mathrm{~Hz}, 3 \mathrm{H}) ;{ }^{13} \mathrm{C} \mathrm{NMR}$ (100 MHz, $\left.\mathrm{CDCl}_{3}\right): 168.8,150.6,146.9,141.3,136.6,131.9,129.5,124.9,21.1,10.2$, 9.2; IR (KBr): 2923, 1778, 1681, 956, 906, $825 \mathrm{~cm}^{-1}$; MS: m/z (\%) $215\left(\mathrm{M}^{+}, 100\right)$, 156 (47); HRMS (EI) Calcd for $\mathrm{C}_{13} \mathrm{H}_{13} \mathrm{NO}_{2}$ : 215.0946. Found: 215.0948.

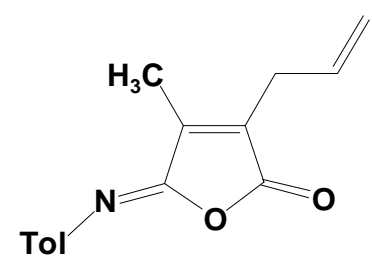




\section{3-allyl-4-methyl-5-p-tolylimino-5H-furan-2-one (13b)}

m.p. 76-78 ${ }^{\circ}$ C. ${ }^{1} \mathrm{H}$ NMR (400 MHz, $\mathrm{CDCl}_{3}$ ): 7.30 (d, $J=8.4 \mathrm{~Hz}, 2 \mathrm{H}$ ), 7.15 (d, $J=8.4$ Hz, 2H), 5.88-5.79 (m, 1H), 5.17-5.11 (m, 2H), 3.18 (d, J = 6.4 Hz, 2H), 2.34 (s, 3H), 2.18 (s, 3H); ${ }^{13} \mathrm{C}$ NMR (100 MHz, $\left.\mathrm{CDCl}_{3}\right)$ : 168.1, 150.4, 147.8, 141.3, 136.7, 133.2, 131.9, 129.5, 125.0, 117.5, 28.1, 21.1, 10.2;IR (KBr): 3031, 2922, 1789, 1681, 1511, 961, 919, $821 \mathrm{~cm}^{-1}$; MS: m/z (\%) $241\left(\mathrm{M}^{+}, 100\right), 79$ (32); HRMS (EI) Calcd for $\mathrm{C}_{15} \mathrm{H}_{15} \mathrm{NO}_{2}$ : 241.1103. Found: 241.1110

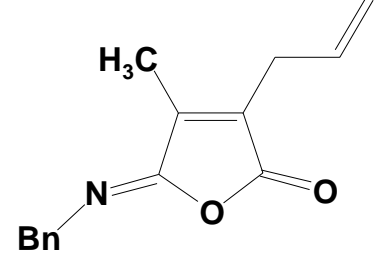

\section{3-allyl-5-benzylimino-4-methyl-5H-furan-2-one (13c)}

m.p. $115-117{ }^{\circ} \mathrm{C} .{ }^{1} \mathrm{H}$ NMR (400 MHz, $\mathrm{CDCl}_{3}$ ): 7.39-7.31 (m, 4H), 7.28-7.24 (m, 1H), 5.91-5.75 (m, 1H), 5.15-5.10 (m, 2H), 4.78 (s, 2H), 3.16 (d, J = 6.0 Hz, 2H), 2.11 (s, $3 \mathrm{H}) ;{ }^{13} \mathrm{C}$ NMR (100 MHz, $\left.\mathrm{CDCl}_{3}\right): 167.6,153.0,147.0,138.7,133.8,131.8,128.5$, 127.9, 127.0, 117.5, 52.8, 28.0, 10.0; IR (KBr): 3041, 2982, 2933, 1778, 1692, 1634 , 1405, 1094, $711 \mathrm{~cm}^{-1}$; MS: m/z (\%) 241 (M+39), 91 (100); HRMS (EI) Calcd for $\mathrm{C}_{15} \mathrm{H}_{15} \mathrm{NO}_{2}$ : 241.1103. Found: 241.1102.

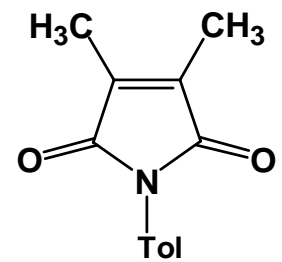

\section{3,4-dimethyl-1-p-tolyl-pyrrole-2,5-dione (14a, See Lit 1)}

m.p. 118-120 ${ }^{\circ} \mathrm{C} .{ }^{1} \mathrm{H}$ NMR (400 MHz, $\mathrm{CDCl}_{3}$ ): 7.26-7.19 (m, 4H), 2.37 (s, 3H), 2.04 (s, 3H); ${ }^{13} \mathrm{C}$ NMR (100 MHz, $\left.\mathrm{CDCl}_{3}\right):$ 171.0, 137.4, 137.3, 129.6, 129.2, 125.7, 21.1, 8.9; IR (KBr): 2922, 1710, 1684, 1516, 1393, 1089, 817, 730, $516 \mathrm{~cm}^{-1}$; MS: m/z (\%) $215\left(\mathrm{M}^{+}, 100\right)$, 156 (55); HRMS (EI) Calcd for $\mathrm{C}_{13} \mathrm{H}_{13} \mathrm{NO}_{2}$ : 215.0946. Found: 215.0945. 


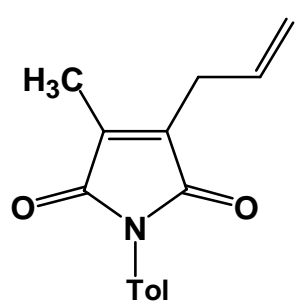

\section{3-allyl-4-methyl-1-p-tolyl-pyrrole-2,5-dione (14b)}

m.p. 59-61 ${ }^{\circ} \mathrm{C} .{ }^{1} \mathrm{H}$ NMR (400 MHz, $\mathrm{CDCl}_{3}$ ): 7.26-7.20 (m, 4H), 5.92-5.83 (m, 1H), 5.19-5.13 (m, 2H), 3.23 (d, $J=6.4 \mathrm{~Hz}, 2 \mathrm{H}), 2.37$ (s, 3H), 2.07 (s, 3H); ${ }^{13} \mathrm{C}$ NMR (100 $\left.\mathrm{MHz}, \mathrm{CDCl}_{3}\right):$ 170.9, 170.4, 138.1, 137.4, 132.2, 129.6, 129.1, 125.7, 125.6, 117.5, 27.8, 21.1, 8.9; IR (KBr): 3085, 3033, 2987, 2923, 1711, 1677, 1514, 1405, 1109, 905, $743 \mathrm{~cm}^{-1}$; MS: m/z (\%) $241\left(\mathrm{M}^{+}, 100\right), 79$ (68); HRMS (EI) Calcd for $\mathrm{C}_{15} \mathrm{H}_{15} \mathrm{NO}_{2}$ : 241.1103. Found: 241.1109.

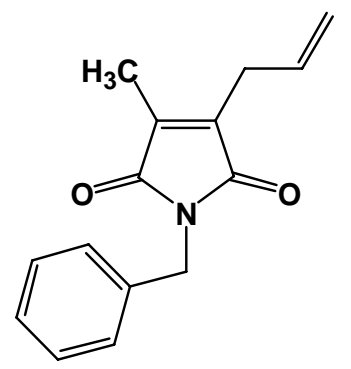

\section{3-allyl-1-benzyl-4-methyl-pyrrole-2,5-dione (14c)}

m.p. 80-82 ${ }^{\circ} \mathrm{C} .{ }^{1} \mathrm{H}$ NMR (400 MHz, $\mathrm{CDCl}_{3}$ ): 7.38 (d, $J=7.6 \mathrm{~Hz}, 2 \mathrm{H}$ ), 7.32-7.24 (m, 3H), 6.56-6.48 (m, 1H), 5.75-5.64 (m, 2H), 4.77-4.67 (m, 2H), 3.32 (d, $J=6.4 \mathrm{~Hz}$, 2H), 2.15 (s, 3H); ${ }^{13} \mathrm{C}$ NMR (100 MHz, $\mathrm{CDCl}_{3}$ ): 177.4, 169.6, 135.8, 134.2, 130.9, 130.6, 128.6, 128.5, 127.9, 127.8, 42.3, 37.6, 16.5; IR (KBr): 3061, 2934, 1695, 1649, 1434, 1170, $702 \mathrm{~cm}^{-1}$; MS: m/z (\%) 241 (M+9 97), 79 (100); HRMS (EI) Calcd for $\mathrm{C}_{15} \mathrm{H}_{15} \mathrm{NO}_{2}$ : 241.1103. Found: 241.1102.

\section{Literature:}

1. Mangaleswaran, S.; Argade, N. P. Synthesis 2002, 7, 865. 


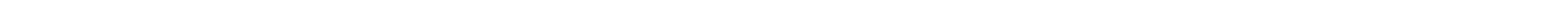




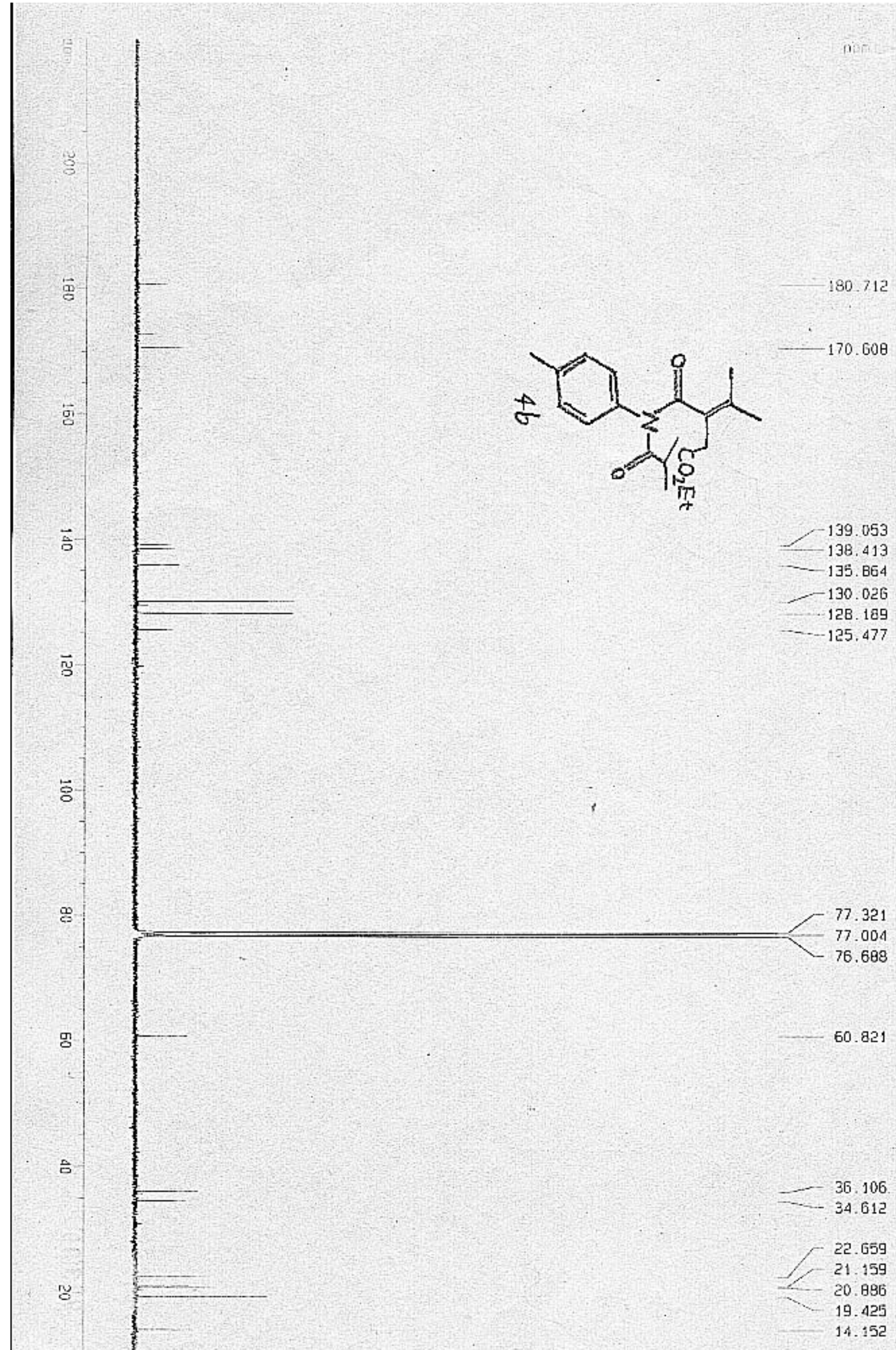




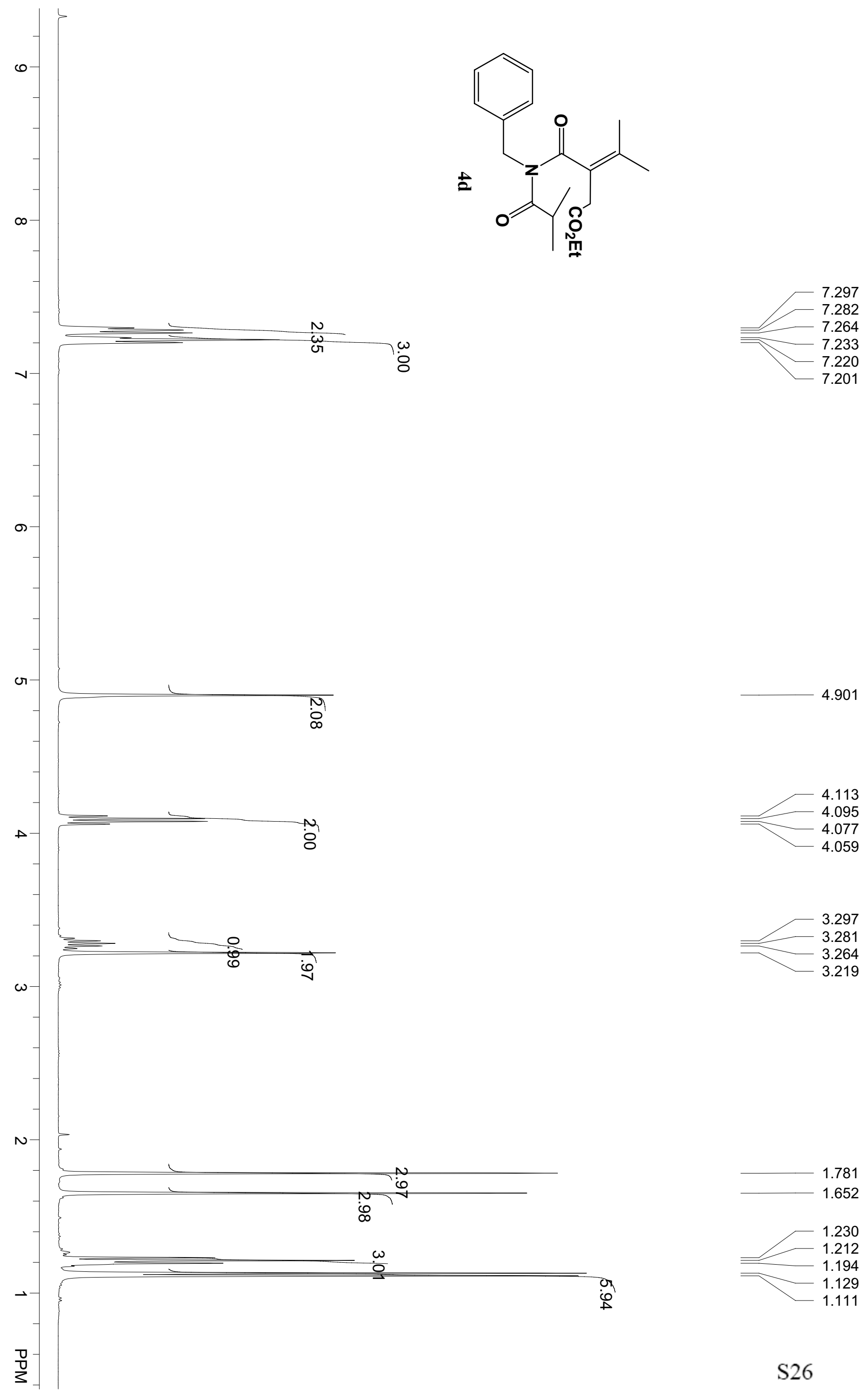




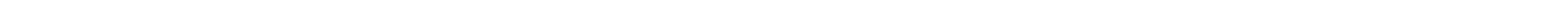




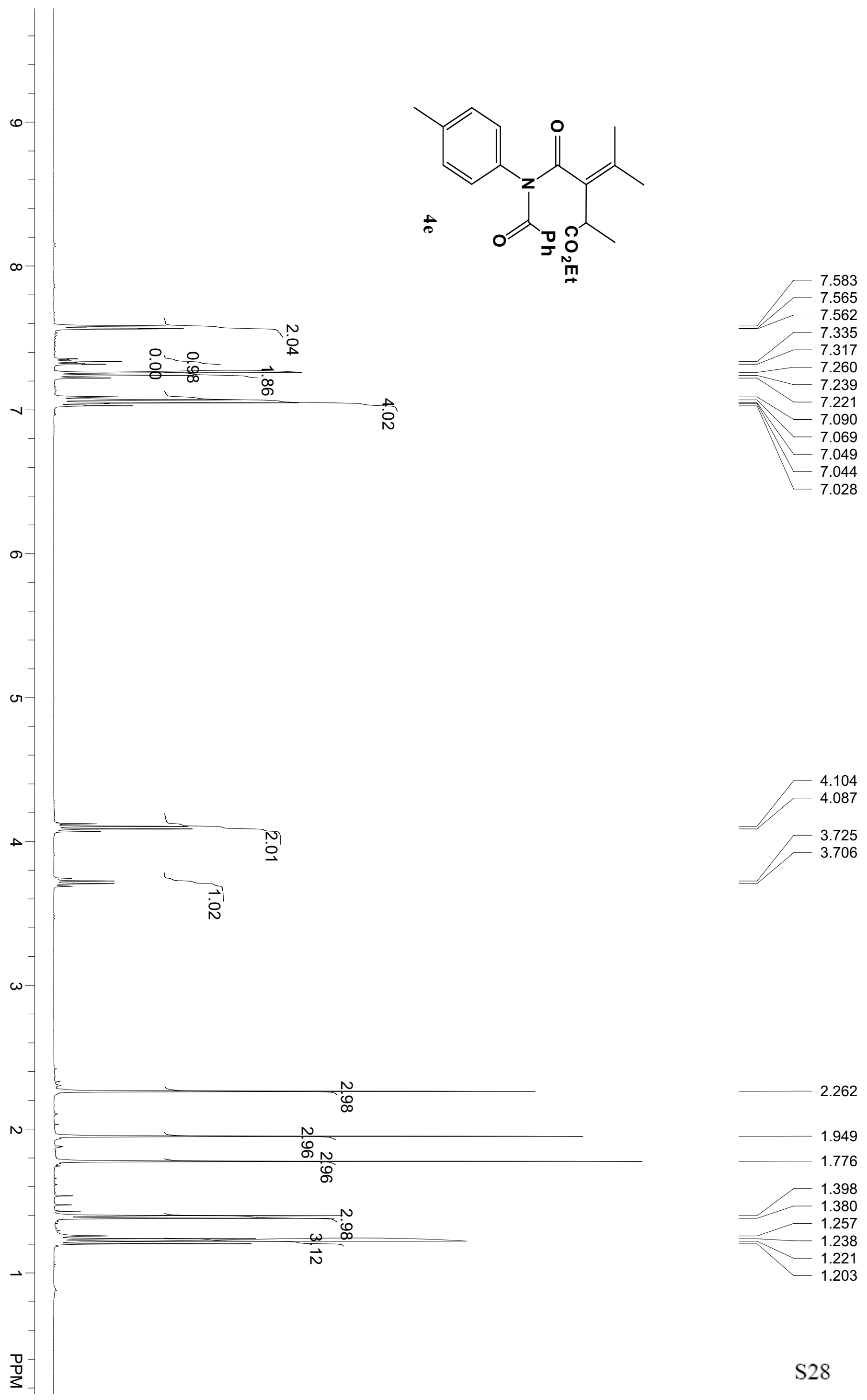




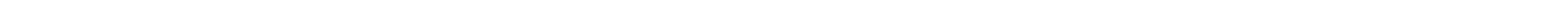




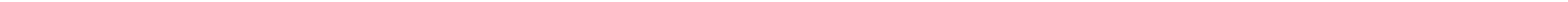




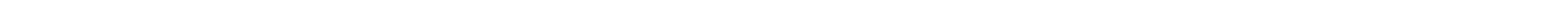



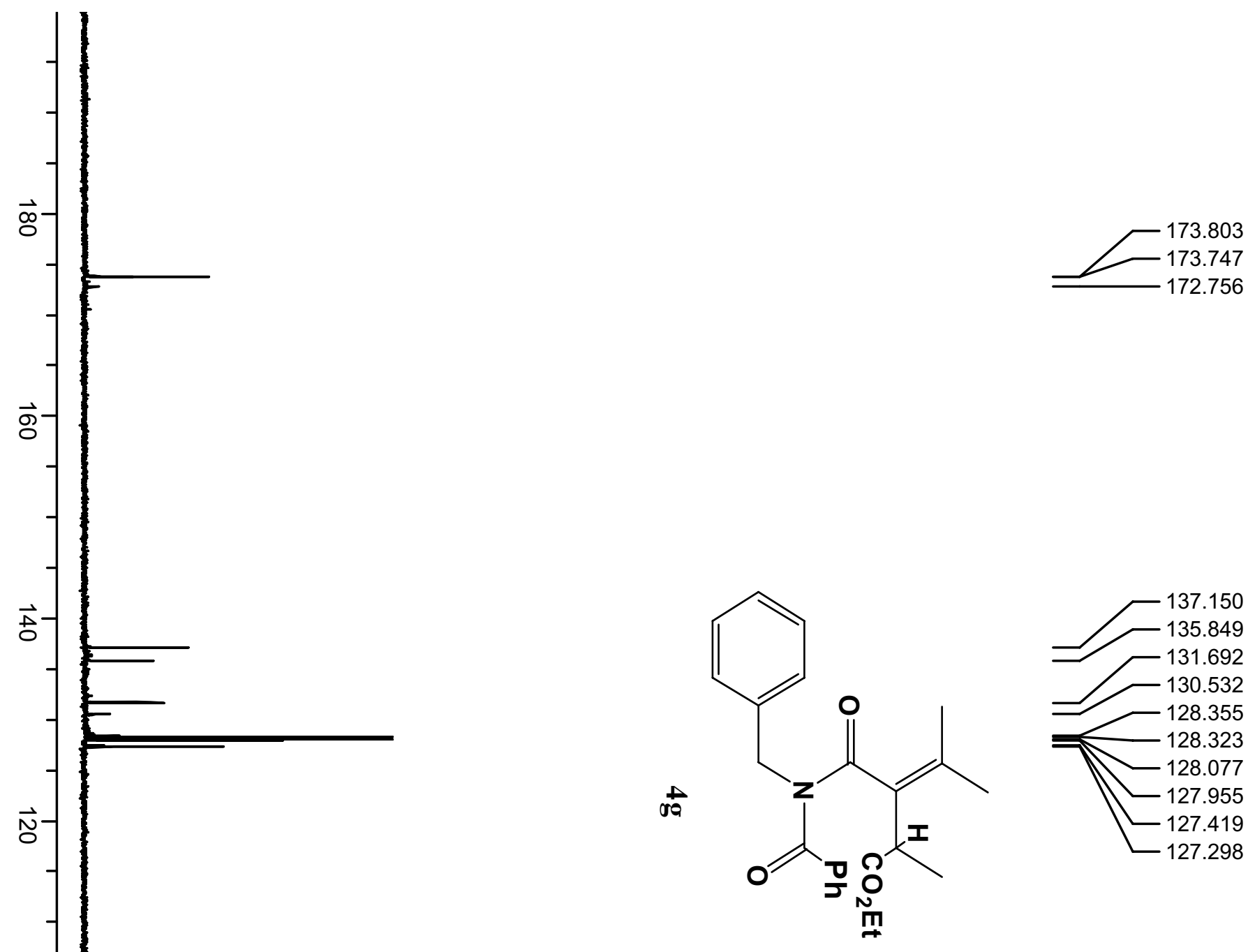

응

ᄋ
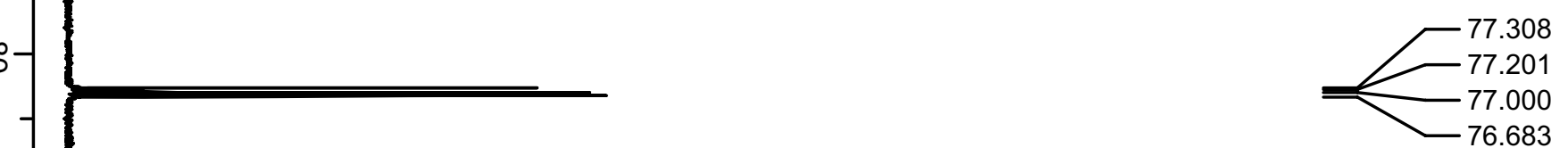

60.709

8
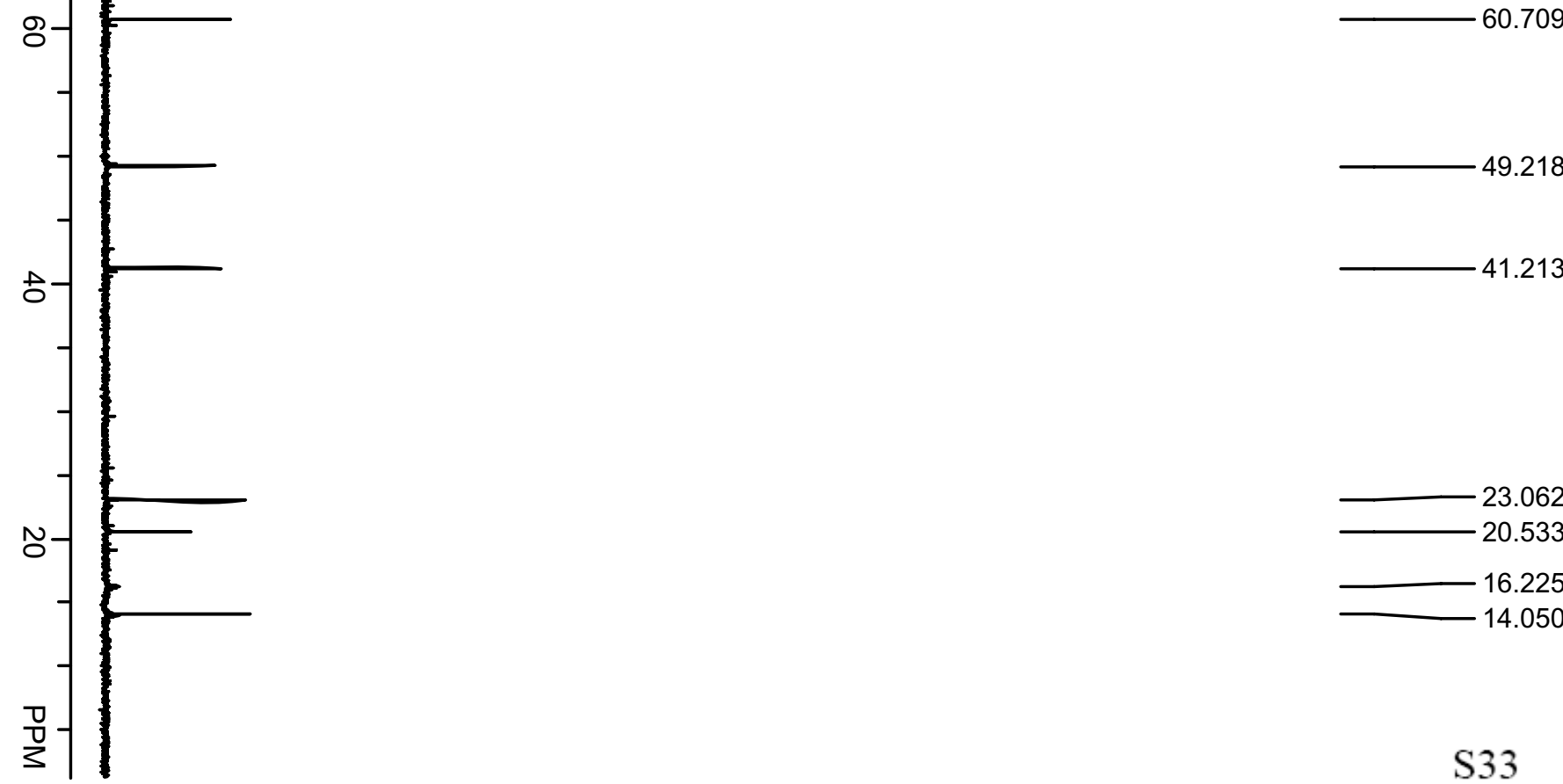

49.218

41.213 


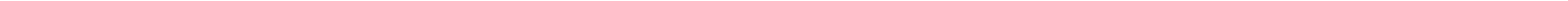




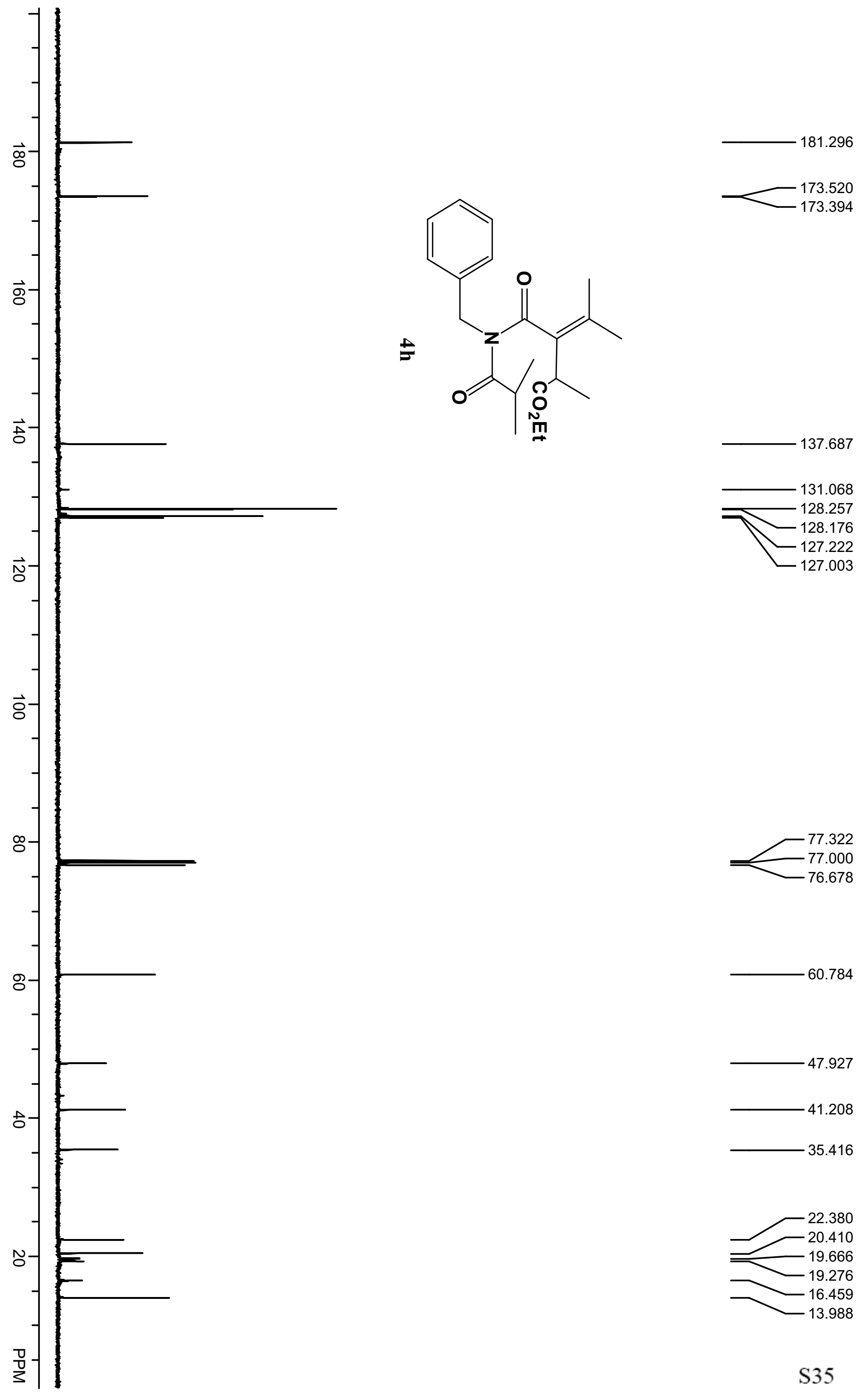




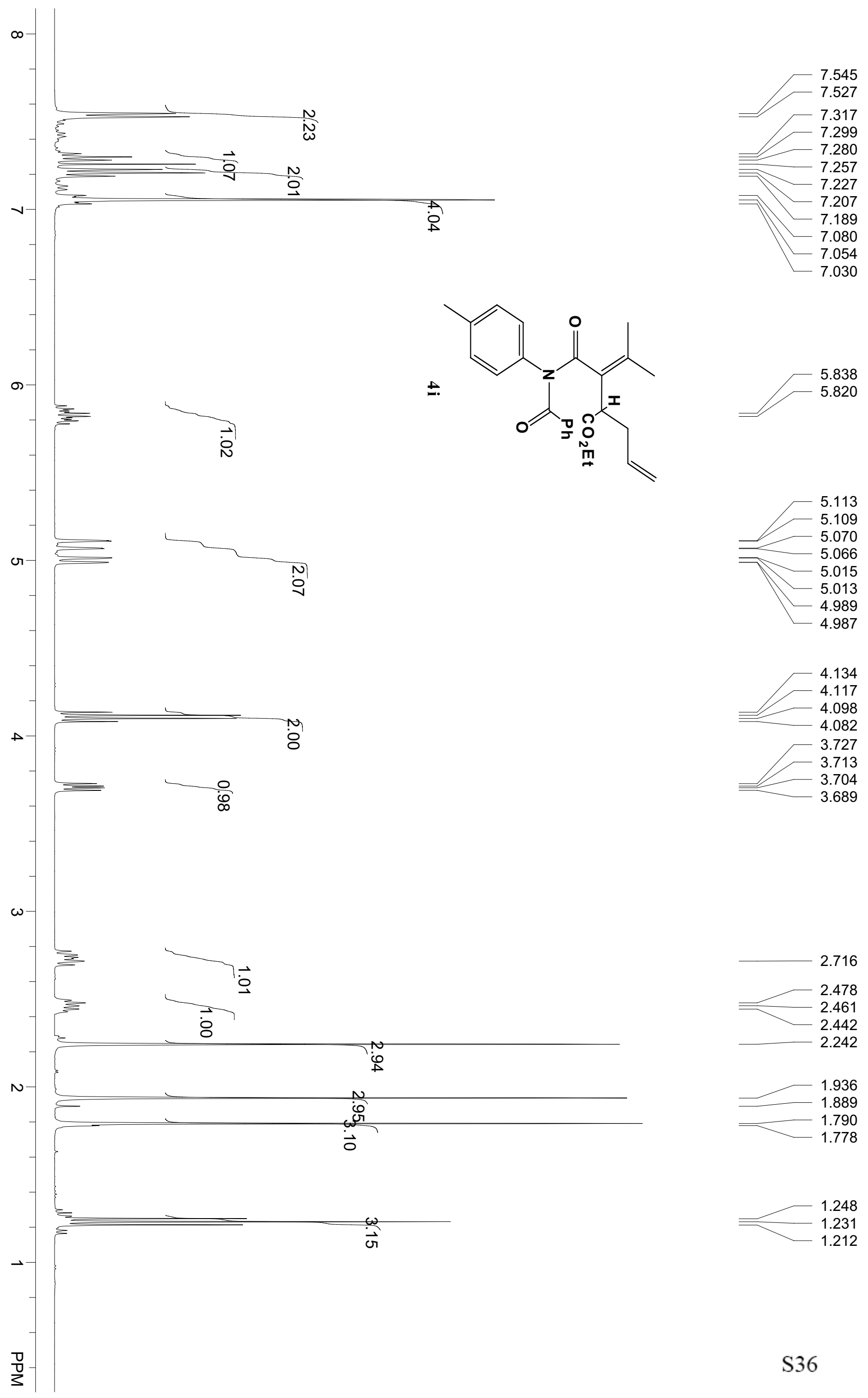




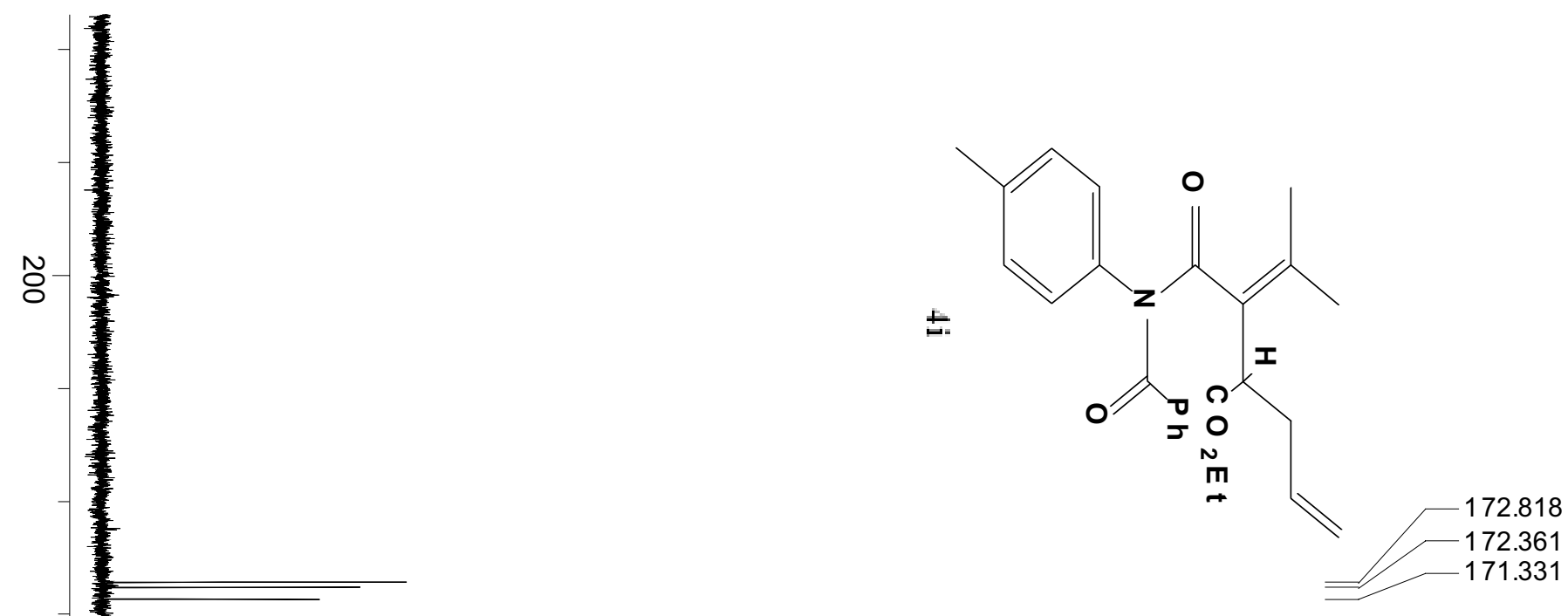


$N$

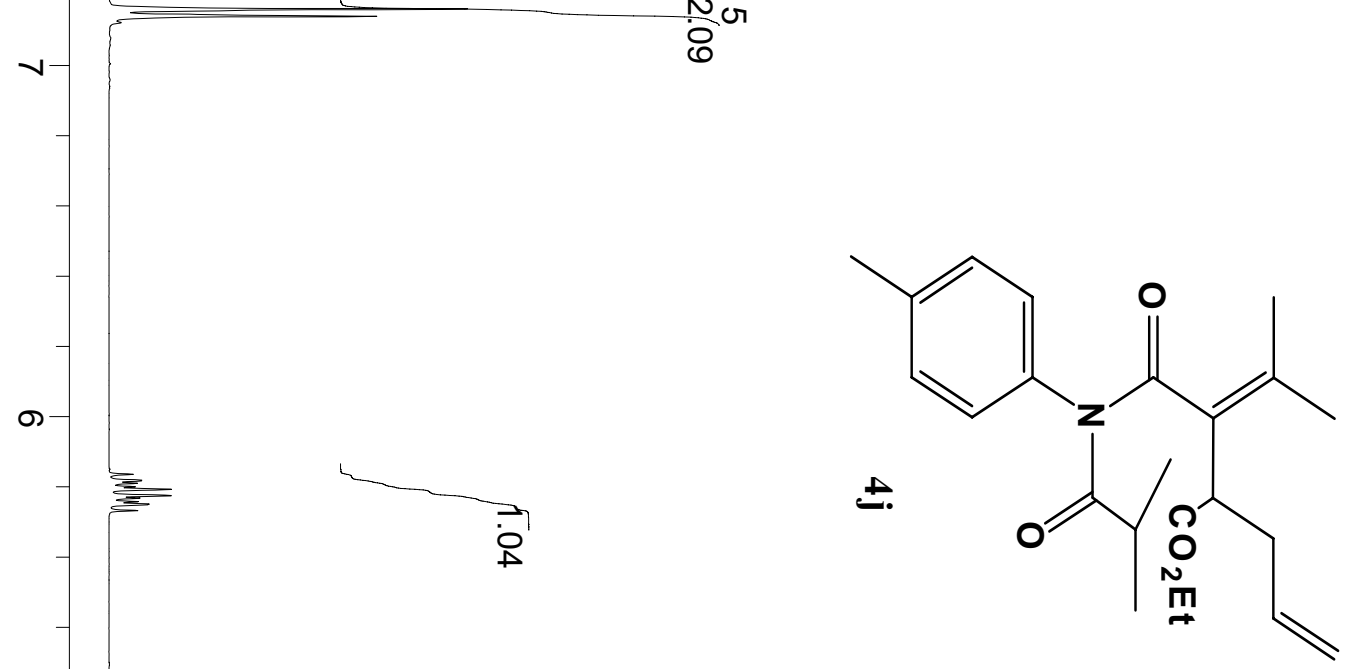

4.119

4.111 4.101 4.093

4.083

4.075

3.709

3.693

3.686

3.670

$\omega$

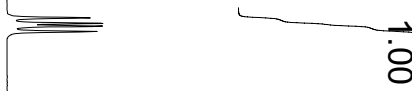

2.576

2.526

2.508

2.492

2.421

2.397

1.785

1.773

$\frac{9}{8}$

1.283

1.266

1.248

1.042

1.025

0.980

w

0.964 


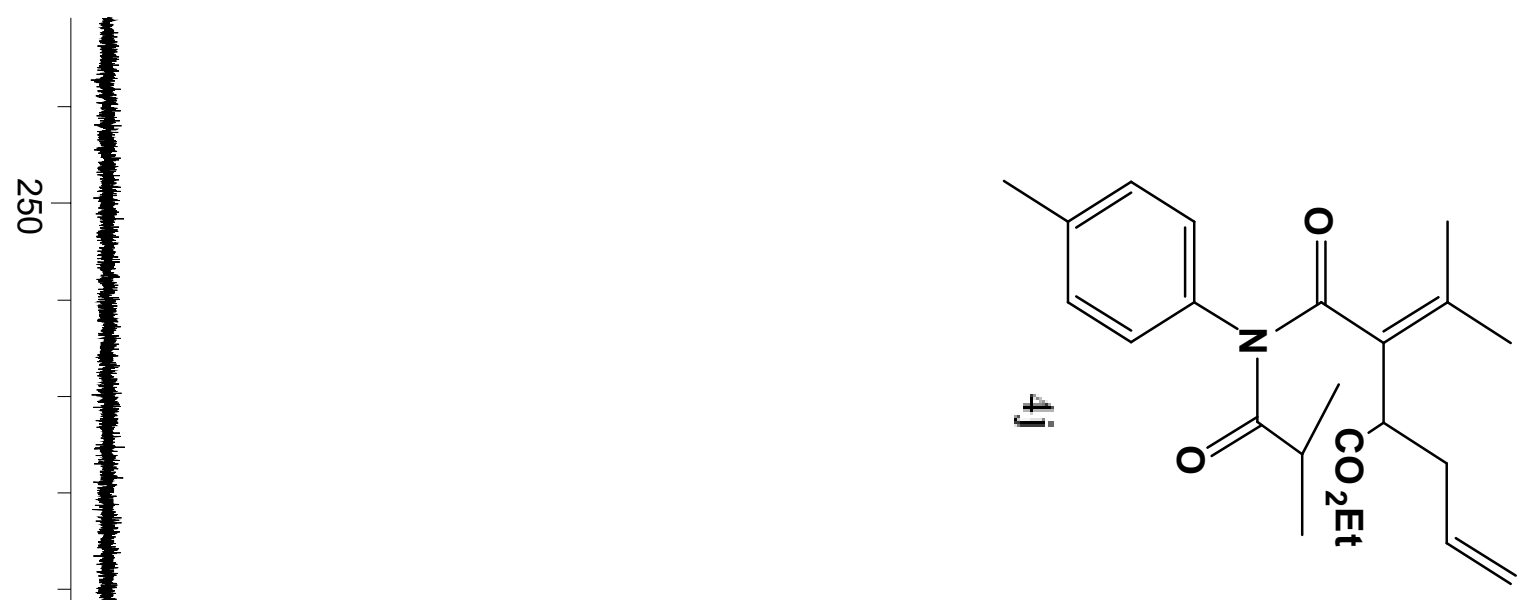

ธั

ज्ञ

음

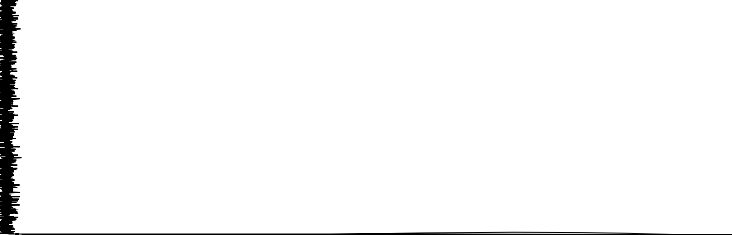

135.942

135.271

130.208

130.183

128.441

128.235

116.788

잉

47.427

36.601

34.063

22118

21.108

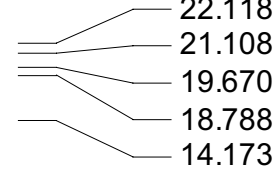




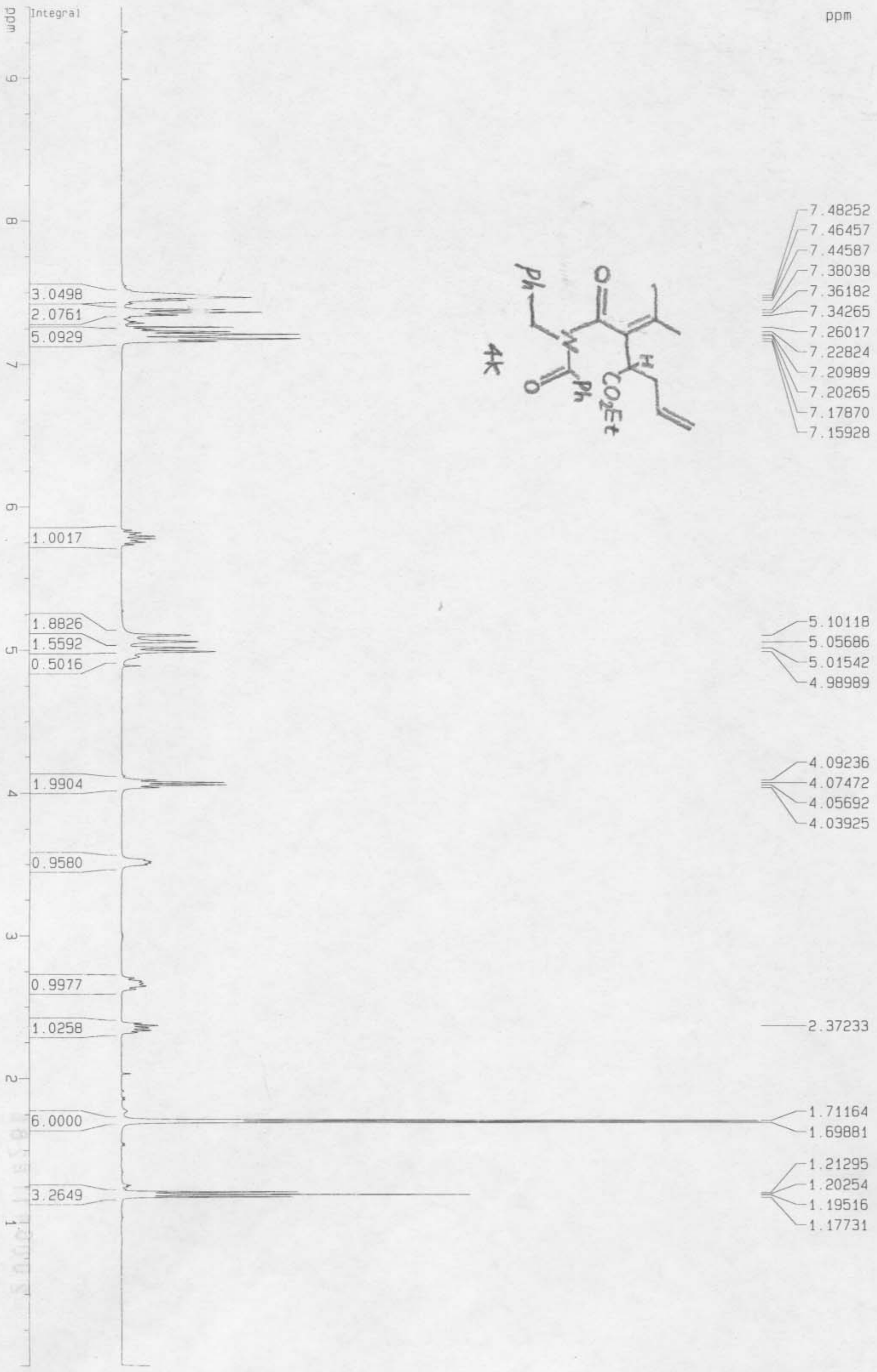




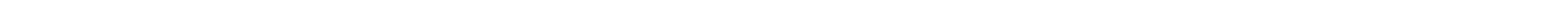




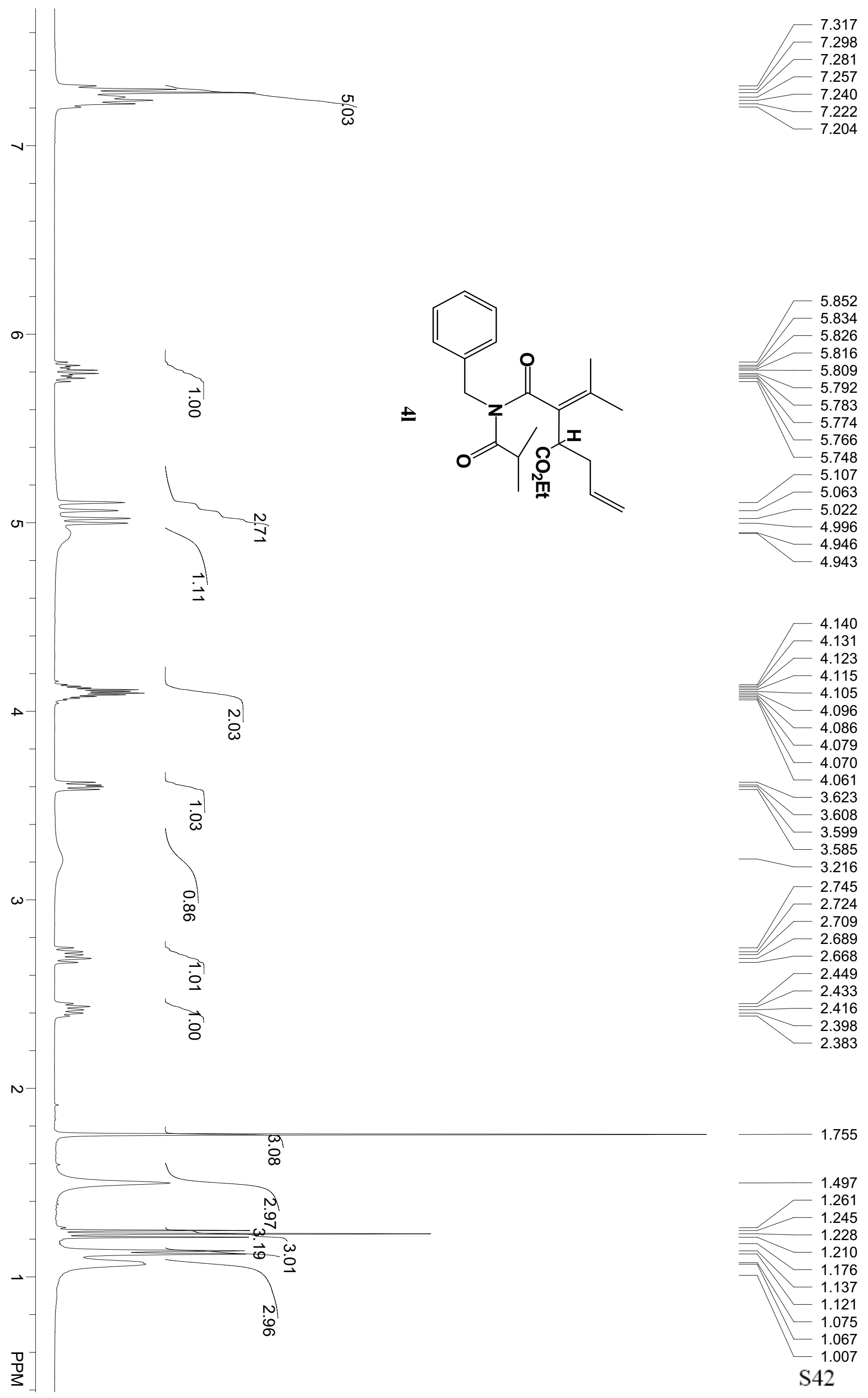




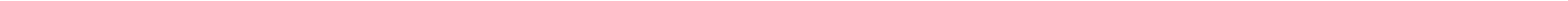




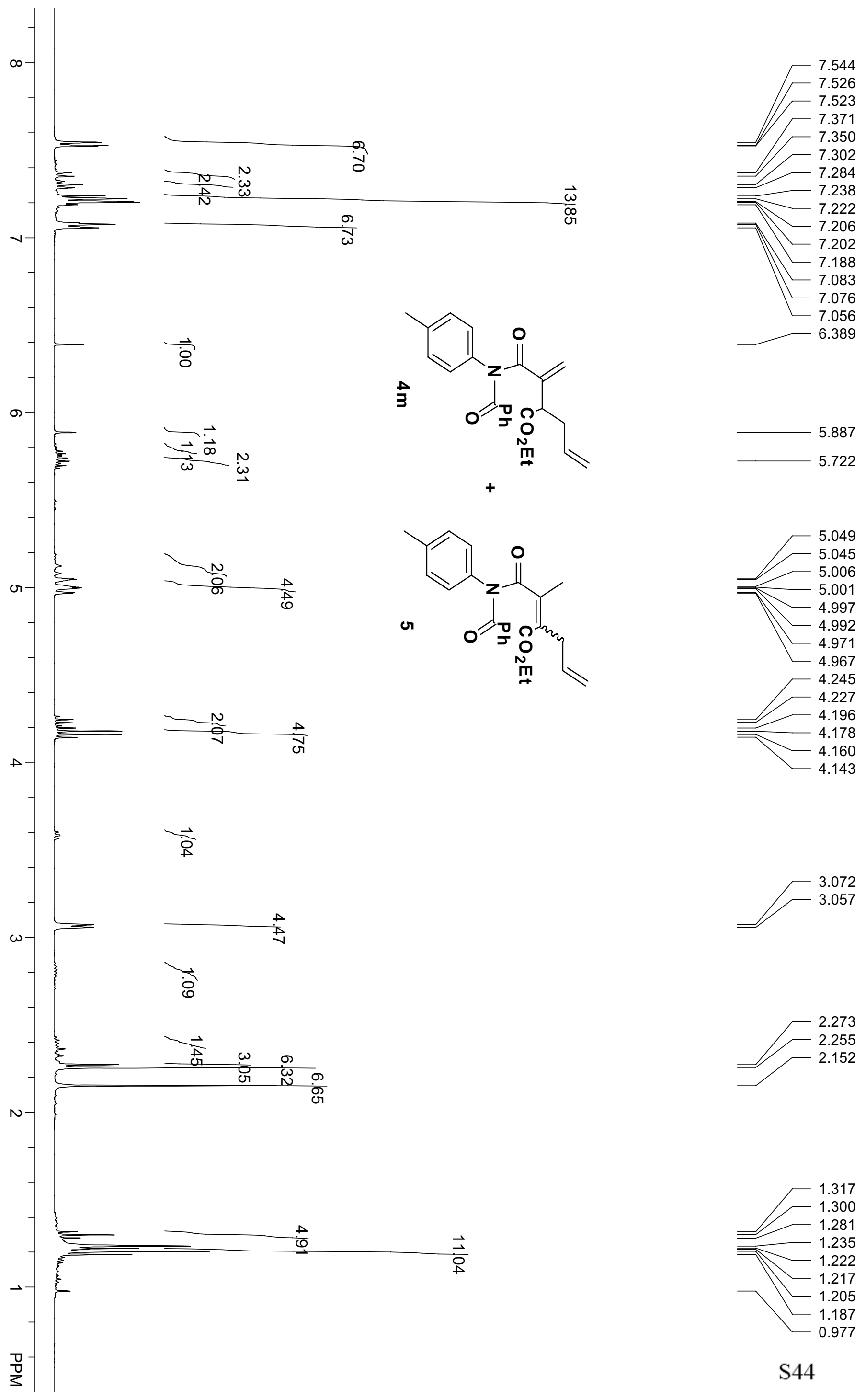




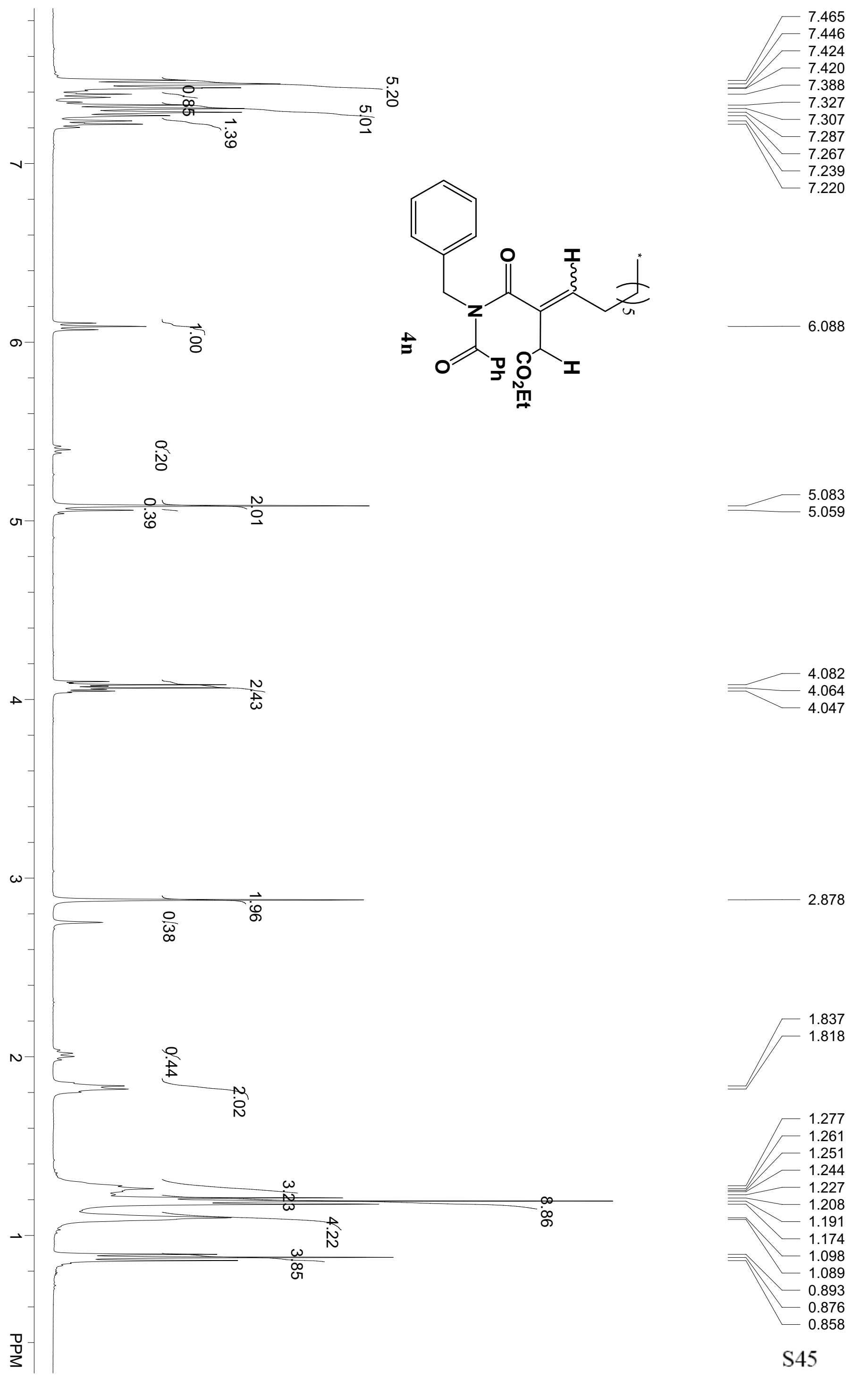




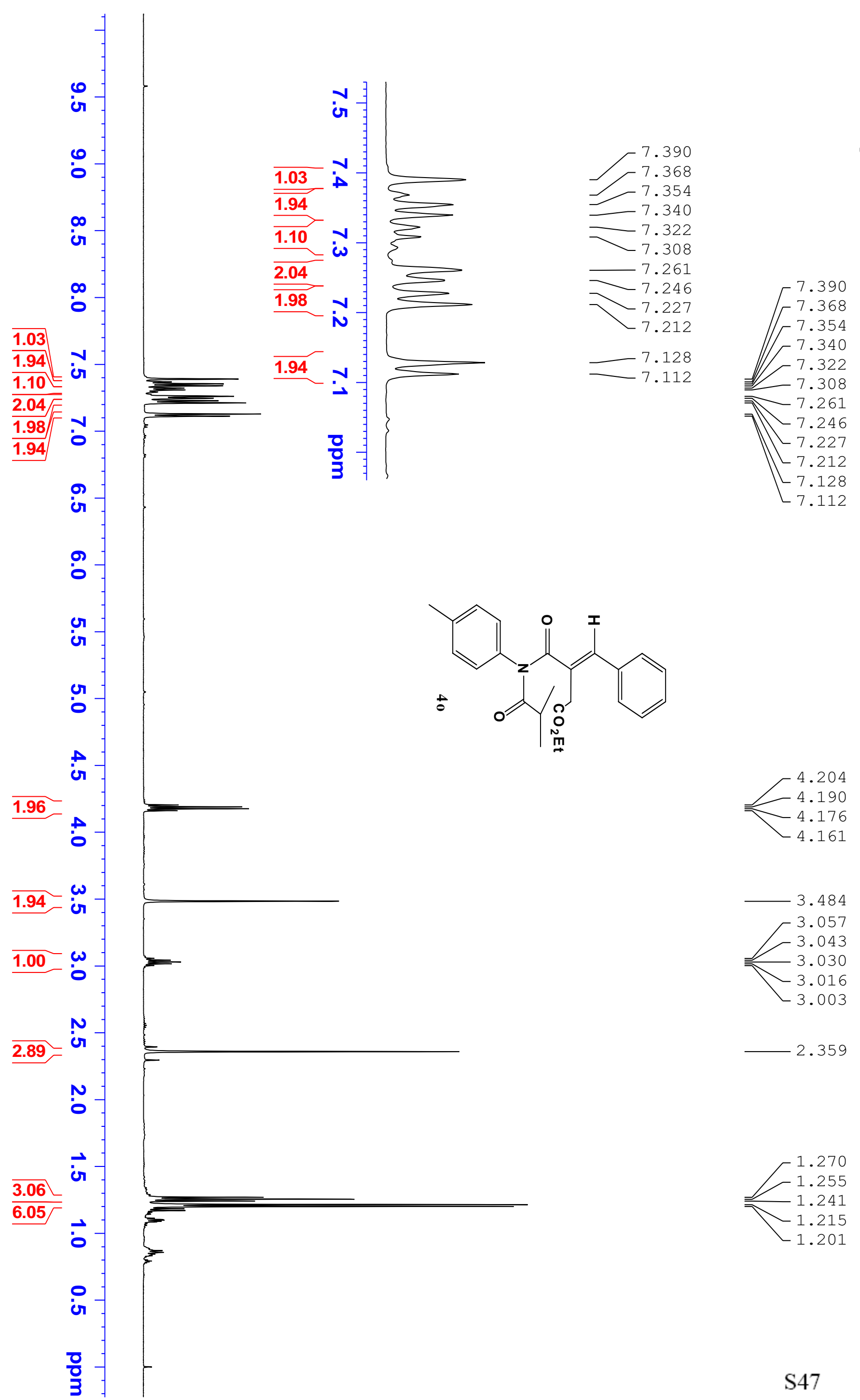




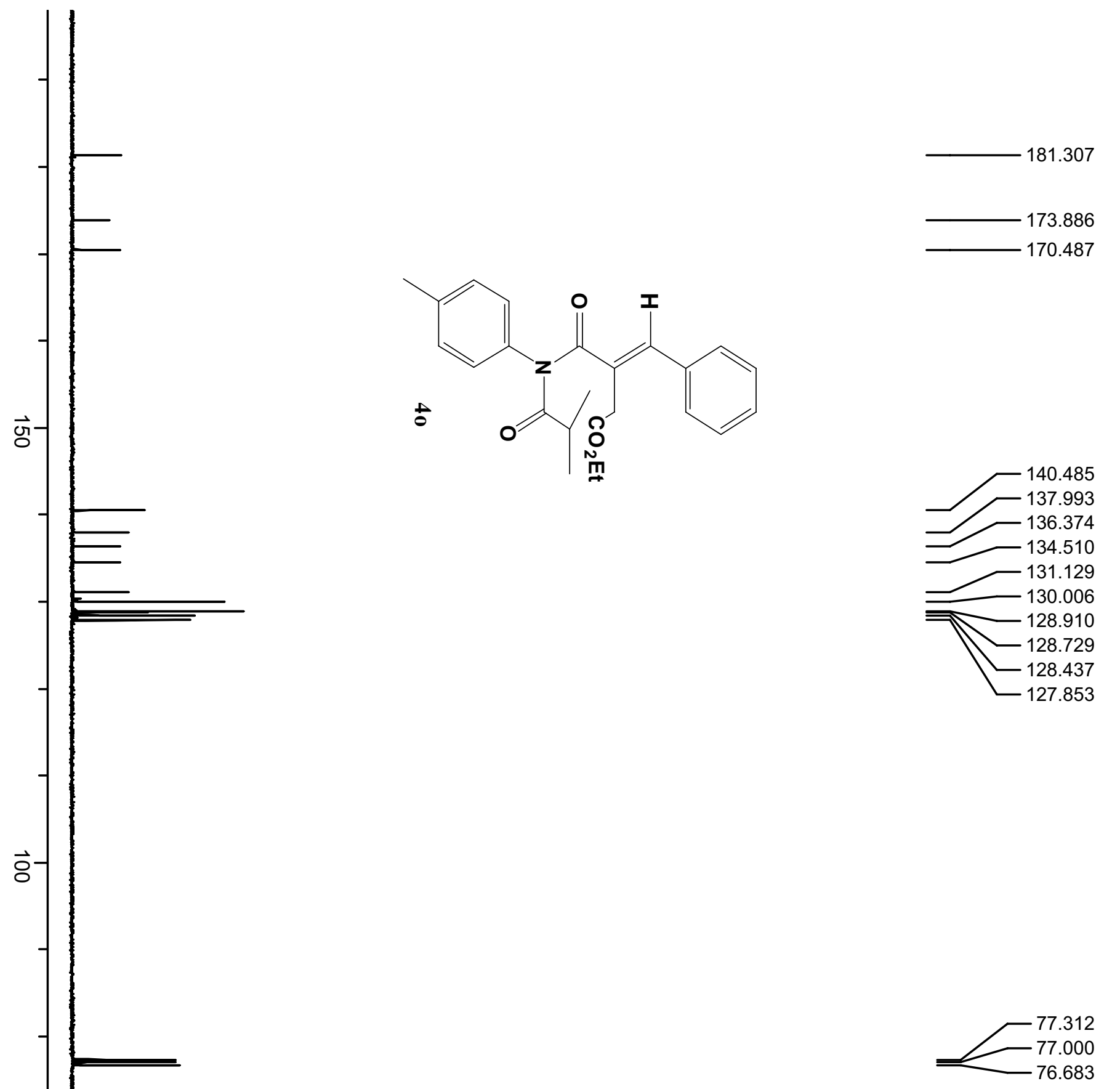




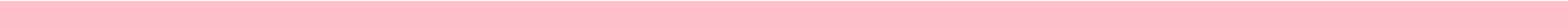


